WIENER SLAVISTISCHES JAHRBUCH, Band 52/2006, 113-134

(C) 2007 by Österreichische Akademie der Wissenschaften, Wien

Stefan Michael Newerkla

\title{
Slavische und slavisierte Toponyme in Österreich am Manhart und unter der Enns
}

\author{
Ergänzende Bemerkungen zu einem neuen Glossar \\ von Etyma eingedeutschter Namen slavischer Herkunft \\ in Niederösterreich
}

Das Institut für Slawistik der Philologisch-Kulturwissenschaftlichen Fakultät der Universität Wien ist die weltweit größte Forschungs- und Lehrstätte für slavische Sprachen, Literaturen und Kulturen. Eine annähernd vergleichbare Breite in Forschung und Lehre weisen lediglich die entsprechenden Institute an der LomonosovUniversität in Moskau und - nur bedingt - an der Humboldt-Universität zu Berlin auf. Mit den Schwerpunkten Sprach-, Literatur- und Kulturkontaktforschung, slavischer Dialektologie einschließlich Burgenlandkroatisch und Slovenisch in Kärnten, slavischer Mediävistik und allgemein der Diachronie hat die Wiener Slavistik ein unverwechselbares Profil und genießt - nicht zuletzt aufgrund seiner thematischen Breite in Forschung und Lehre - international höchstes Ansehen. Auf mehreren Fachgebieten gehört es dabei zu den weltweit führenden; unzweifelhaft trifft dies etwa auf die historische und vergleichende slavische Sprachwissenschaft zu. In deren Rahmen bildet traditionell die Erforschung der im österreichischen urkundlichen und onomastischen Material belegten Namen des mittelalterlichen Slaventums in Österreich einen wichtigen Schwerpunkt. Am Wiener Institut für Slawistik ist dieser Forschungsbereich vor allem mit den Namen Otto Kronsteiner (insbesondere in den 70er und 80er Jahren des 20. Jahrhunderts) und Georg Holzer (ab den 90er Jahren des 20. Jahrhunderts bis in die Gegenwart) verbunden. Er war auch der Leiter des vom Fonds zur Förderung der wissenschaftlichen Forschung (FWF) finanzierten, zweijährigen Forschungsprojekts „Die Sprache des mittelalterlichen Slaventums in Österreich“, das seine institutionelle Heimat in der BalkanKommission der Österreichischen Akademie der Wissenschaften gefunden hatte. Das Forschungsvorhaben „Das slavische Substrat in Österreich aus linguistischer Sicht" war an dieser ja bereits zuvor etabliert worden. 
Die Arbeiten an dem genannten Projekt führte die junge Wiener Slavistin und Mitarbeiterin der Österreichischen Akademie der Wissenschaften Angela Bergermayer durch. In Abstimmung mit Georg Holzer wurde dabei die Idee der wörterbuchmäßigen Erfassung der im österreichischen urkundlichen und onomastischen Material belegten Namen des mittelalterlichen Slaventums in Österreich unter Hinzufügung von urkundlichen Nennungen und Vergleichstoponymen in den slavischsprachigen Ländern realisiert. Das erstellte Glossar legte das wirkliche Mitglied Radoslav Katičić in der Akademiesitzung am 25. Juni 2004 vor; es steht seit vergangenem Jahr der interessierten Öffentlichkeit in der vom Verlag der Österreichischen Akademie der Wissenschaften gewohnten hochqualitativen Ausführung als gedrucktes Buch zur Verfügung: Angela Bergermayer, Glossar der Etyma der eingedeutschten Namen slavischer Herkunft in Niederösterreich (= Schriften der Balkan-Kommission 44), Wien (Verlag der Österreichischen Akademie der Wissenschaften) 2005, 368 S. Mit dem vorliegenden Glossar ist ein weiterer wichtiger Mosaikstein dem Feld der Forschungen zum untergegangenen Slaventum in Österreich hinzugefügt worden, der aus slavistischer Sicht in einer Reihe mit Monographien wie Kronsteiner 1981 oder Holzer 2001 genannt werden kann.

Dem eigentlichen Wörterbuchteil geht nach einem kurzen Vorwort (Bergermayer 2005: 7-8) eine klar verständliche und in ihrem Umfang durchaus angemessene Einleitung (9-20) voraus, die allgemeine Vorbemerkungen zum untergegangenen Slaventum in Österreich, zur bisherigen Forschungs- und Quellenlage sowie zur thematischen und stofflichen Eingrenzung des Glossars (9-14), zum eigentlichen Aufbau des Glossars (14-19) sowie zu den Indizes (19-20) enthält.

Das für die Anordnung der Namen im Glossar und in den Indizes verwendete Alphabet wird mehr oder weniger kommentarlos angeführt (14). Das erschwert jedoch die Rezeption des Buches durch interessierte Laien, aber auch Wissenschaftler anderer Wissenschaftsdisziplinen (wie allgemeine Geschichte, Siedlungs- und Sozialgeschichte, Archäologie, Onomastik, germanistische Sprachwissenschaft, Geschichte und Geographie der deutschen Mundarten), die laut Autorin von ihrem Buch profitieren sollen (11) und dies zweifellos könnten. In der vorliegenden Version wird aber nur der Eingeweihte verstehen, worin z. B. die nicht zu vernachlässigenden Unterschiede zwischen $e, \dot{e}, \grave{e}, e, \ddot{e}, \dot{e}$ bzw. $l, l^{\prime}, l, l$, (14) liegen. Hier wurde also vom eigenen Wissensstand ausgegangen, ohne zu berücksichtigen, dass die Ausführungen auch von fachnahen Kollegen verstanden werden sollten. Dazu kommt noch der Hang der Autorin zu komplizierten Satzkonstruktionen, wodurch die rasche Rezeption von an und für sich einfachen Sachverhalten unnötig behindert wird, z. B.:

Auf diese Weise soll die Wortbildung bzw. die Morphologie der Namen klar ersichtlich gemacht und, indem auch Ableitungsbasen als Lemmata geführt werden, die nicht selbst, sondern nur in Ableitungen als Namen belegt sind, nichtsdestoweniger damit aber in der Regel als in der Sprache existent vorausgesetzt werden dürfen, der im Namenmaterial dokumentierte Wortschatz des untergegangenen Slavischen möglichst vollständig erfasst werden. (15)

oder:

Auf eine entsprechende Dreiteilung des Glossars (1. Glossar sicherer slavischer Basen, 2. Glossar eventueller slavischer Basen, 3. Glossar slavisierter Basen) wurde in Übereinkunft mit dem Projektleiter verzichtet, weil sie voraussetzt, dass der Benützer schon von vornherein weiß, ob es $\mathrm{zu}$ dem, was er sucht, eine sichere slavische, eine eventuelle slavische oder eine slavisierte Basis gibt, oder aber zur Folge hat, dass er an drei Stellen des Buches nachschlagen muss. (16) 
An dieser Stelle sei bemerkt, dass letzter Satz auch inhaltlich zu hinterfragen ist. Dank der ausführlichen Indizes im Anschluss an das Glossar wird ein interessierter Leser ohnehin zuerst in den Wortlisten suchen, da er in den meisten Fällen nicht in der Lage sein wird, auf Anhieb die slavische Ausgangsform von eingedeutschten Namen richtig zu rekonstruieren. Die angedachte Dreiteilung hätte also durchaus erfolgen können und erschiene mir als eine wertvolle Bereicherung.

Für jeden, der sich bereits einmal mit der vorgestellten Problematik auseinander gesetzt hat, ist das folgende Glossar (21-304) eine Fundgrube. Solche Leser werden gleichzeitig auch auf Vieles stoßen, was ihnen bereits aus anderen Regionen mit ähnlicher Siedlungsgeschichte geläufig ist. Die Konzeption des Wörterbuchs ist durchdacht und um maximale Transparenz bemüht, so dass ihm ein hoher Grad an Benutzerfreundlichkeit zuzugestehen ist. Das dargereichte Wörterverzeichnis erfasst auf 284 Seiten über 750 Lemmata (einschließlich aller Ableitungsschritte) in Wortneststruktur und gibt so Auskunft über rund 1000 eingedeutschte geographische Namen und Personenbenennungen slavischer Herkunft in Niederösterreich sowie über rund 1750 Vergleichsetyma außerhalb Niederösterreichs. Die einzelnen Wörterbuchartikel werden von *aworb 'Ahorn, Spitzahorn, Bergahorn' (21-22) bis *Županъ 'Vorsteher, Art Dorfältester' (302-304) in alphabetischer Reihenfolge der Lautung zum Entlehnungszeitpunkt angeführt und sind sehr übersichtlich aufgebaut. Als Namen belegte Etyma schreibt Bergermayer mit großem Anfangsbuchstaben. Ableitungen stehen unter der jeweiligen Ableitungsbasis; pro Ableitungsschritt wird dabei ein Geviertstrich (-) vorangestellt. ${ }^{1}$ Danach erfolgen jeweils Bedeutungserklärungen zum Lemma und etymologisch entsprechende Appellative und Personennamen in (modernen) slavischen Sprachen, vor allem im Slovenischen und Tschechischen (das Slovakische als ebenfalls direkt angrenzende Slavine wird leider in den meisten Fällen nicht berücksichtigt bzw. wird auch die entsprechende onomastische Literatur überraschenderweise kaum berücksichtigt). Innerhalb des Lemmas werden chronologisch nach dem Datum des Erstbelegs die betreffenden eingedeutschten Namen samt ihren Belegen angeführt, wobei die einzelnen slavischen Benennungen jeweils mit einer eigenen Nummer versehen werden. Die Quellenangaben sind den Belegen hinzufügt. Meist folgt darauf ein kurzer namenkundlicher bzw. sprachhistorischer Kommentar über die Entlehnungszeit und die lautliche Entwicklungsstufe im Slavischen, bevor am Ende der Angaben zu den einzelnen Namen innerhalb eines Lemmas wichtige Sekundärliteratur genannt wird. Abschließend wird noch auf Vergleichsnamen außerhalb Österreichs hingewiesen, ohne Vollständigkeit anzustreben. Die Etymologien und Rekonstruktionen sind durchwegs plausibel und sorgfältig erstellt; in umstrittenen Fällen erscheinen sie zumindest als möglich rekonstruiert, wenn auch nicht immer richtig (z. B. die versuchte Herleitung von Göllitzhof < slav. *Golica, 81-82; dazu näher unterhalb).

Nach dem Abschnitt mit der Auflösung der Abkürzungen (305-330), einem Literatur- und Quellenverzeichnis, in dem vermischt Quellen, Literatur und auch die Abkürzungen für Karten angeführt werden (305-328) sowie der Liste allgemeiner Abkürzungen und Zeichen (328330) folgen zum Abschluss noch die für die Erschließung des Glossars wichtigen und aussagekräftigen Indizes (331-366), ein rückläufiger Index der rekonstruierten mittelalterlichen slavischen Namen (331-335), ein Index von Appellativen abgeleiteter slavischer Ortsnamen (336-338), ein Register slavischer Personennamen und von Personennamen abgeleiteter slavischer Ortsnamen weiter unterteilt in Vollnamen und Kurznamen (338-340), ein Index heutiger (amtlicher) und abgekommener eingedeutschter geographischer Namen und Personenbenennungen (341-348), ein Register slavischer Vergleichstoponyme außerhalb Niederösterreichs (348-363) und eine regionale Zuordnung der mittelalterlichen slavischen Namen nach ÖK-Nummern (363-366). Am Ende der Publikationen befindet sich eine Abbildung von Niederösterreich (und Wien) mit der Blattübersicht der österreichischen Karte (ÖK) im Maßstab 1:50 000 (unpaginiert [367]).

Nicht zuletzt aufgrund seines gut erschlossenen Glossars wird das Buch von Angela Bergermayer fortan als wichtige Quelle für die weitere Erforschung der Etyma slavischer Her-

${ }^{1}$ Personennamen werden allerdings immer als Ableitungsbasis der ersten Stufe geführt. 
kunft in Niederösterreich wertvolle Dienste leisten, gerade weil diese Forschung noch lange nicht als abgeschlossen bezeichnet werden kann. Neben der Erwähnung dieser zahlreichen Positiva sind aber auch einige allgemein gehaltene Kritikpunkte vorzubringen, die vor allem als ergänzende Bemerkungen zu diesem neuen Wörterverzeichnis im Hinblick auf eine eventuelle Neubearbeitung des Stoffes verstanden werden sollen und somit den Rahmen einer klassischen Rezension sprengen.

Zunächst ist festzustellen, dass Bergermayer (2005: 11) von Niederösterreich im historischen (Wien einschließenden) Sinn sprechen möchte. Dabei vergisst sie jedoch, dass das historische Land Niederösterreich neben dem unbewohnten Thayadreieck zwischen Thaya und March im Umfang von ca. $30 \mathrm{~km}^{2}$ (früher östlicher Teil der Gemeinden Hohenau an der March/Cahnov und Rabensburg/Ranšpurk, heute Teil des Gemeindekatasters von Lanžhot/Landshut) auch das gesamte Gemeindegebiet von Feldsberg ${ }^{2}$ (heute Valtice, damals Valčice) im Umfang von $87 \mathrm{~km}^{2}$ (10 936 Ew.) mit den Ortschaften Garschönthal (vormals auch Garšentál, heute Úvaly), Oberthemenau (heute Charvátská Nová Ves), Unterthemenau (heute Poštorná) und Bischofswarth (heute Hlohovec) sowie 13 Gemeinden bei Gmünd (Cmunt) im Umfang von insgesamt $113 \mathrm{~km}^{2}$ (ca. 12000 Ew.) eingeschlossen hat, die nach dem Vertrag von Saint-Germain-en-Laye, unterzeichnet am 10. 9. 1919, mit Stichtag 31. 7. 1920 an die Tschechoslovakei abgetreten werden mussten. Im damaligen Gerichtsbezirk Gmünd (Cmunt) waren dies die Ortsgemeinden Beinhöfen (heute Ortsteil Německé von Dvory nad Lužnicí), Erdweis-Sofienwald (vormals Hlinná, heute Nová Ves nad Lužnicí und Žofina Hut'), Naglitz (heute Nakolice), Schwarzbach (vormals auch Švarcbach, heute Tušt'), Tannenbruck-Thiergarten (heute Ortsteil Trpnouze von Hranice und Obora), Weißenbach (heute Vyšné) und Witschkoberg (heute Halámky) zur Gänze, Böhmzeil-Josefschlag (heute als Straßenzug Česká Cejle in Richtung Zollhaus Gmünd bzw. als Žižkovo předměstí, vormals Josefovsko, Stadtgebiet von České Velenice) zu $5 / 6$, Zuggers (heute Krabonoš)-Breitensee (Bratisej) zu $2 / 3$ und Wielands (Velenice)-Unterehrendorf-Oberehrendorf (ein Teil von Unter-Wielands/Dolní Velenice, vormals auch Bělenec bzw. Běleč, gehört heute ebenfalls zu České Velenice) zur Hälfte betroffen; im damaligen Gerichtsbezirk Schrems (Skřemelice) die Ortsgemeinde Gundschachen (heute Kunšach) zur Gänze, Rottenschachen (heute Rapšach) zu $4 / 5$ und Brand-Nagelberg (Lomy) zu $1 / 5$ sowie im damaligen Gerichtsbezirk Weitra (Vitoraz) die Ortsgemeinde Höhenberg-Reinpolz zu $1 / 4$ (AKNÖ 1864 ff., Brunner 1937: 428-429, Garscha 1995: 71, Gutkas 1990: 347). Die Namen dieser Gemeinden fehlen bis auf Garschönthal (Bergermayer 2005: 84) leider auch im Glossar, obwohl es sich bei einigen zweifellos um eingedeutschte Namen slavischer Herkunft handelt.

Gerade für Niederösterreich, in dem bis zum Ende der Monarchie eine nicht zu vernachlässigende Anzahl von slavischsprachigen Tschechen, Mährern, Slovaken und Kroaten auch außerhalb Wiens sesshaft war (Šembera 1844 f., 1868, 1877), ist

\footnotetext{
${ }^{2}$ In Kursivdruck stehen in der Folge alle - aus heutiger Sicht - Exonyma.
} 
eine ganze Reihe von heute zum Großteil bereits außer Gebrauch gekommenen slavischen, insbesondere tschechischen (weniger slovakischen und kroatischen) Exonymen belegt, vor allem für die Viertel nördlich der Donau. ${ }^{3}$ Es ist sonderbar, dass diese bis auf č. Dyje für den Fluss Thaya (Bergermayer 2005: 74) und č. Lužnice für die Lainsitz (ibd.: 141-142) nicht einmal als Vergleichsnamen unter den jeweiligen Lemmata angeführt werden, u. a. fehlen etwa č. Biteš für Vitis (*Byti/ıšb, 50-51); č. Čistějov für Zistersdorf (*Čbstějb, 58); č. Drozdovice für Drosendorf (*Droznъ, 70-71); č. Jer(o)uš für Geras (*Jerošb/*Jerušb, 106), č. Ličov für Litschau (*Ličowb, 133); č. Pulkava für Pulkau (*Pъlkawa, 199-200); č. Re(j)c für Retz (*Rěcě, 221-222; gebräuchlich war auch č. Re(j)csko für das Retzer Land); č. Skřemelice für Schrems (*Skremenınica/*Skremenica, 235); č. Světlá für Zwettl (*Swětblb, 257-258) u. v. a. m. (vgl. die Ortsnamenkonkordanzen am Ende dieses Beitrags).

Was das Quellenmaterial für die Darstellung des slavischen Substrats im ehemals slavisch besiedelten Niederösterreich angeht, wäre eine breitere kritische Auseinandersetzung mit allen bereits zu diesem Thema vorliegenden Arbeiten notwendig gewesen. Aus westslavistischer Sicht vermisst man etwa völlig die objektive Einarbeitung des umfassenden, auch onomastischen Materials, das wir schon beim angesehenen Rechtsanwalt, Historiker, Ethnographen, Philologen und ab 1849 auch Professor für Bohemistik am neu eingerichteten Institut für Slawistik Alois Vojtěch Šembera (1807-1882) finden. So verfasste der in Vysoké Mýto (Hohenmauth) geborene, vormalige Tschechischlehrer an der Ständeakademie in Olomouc (Olmütz) unter anderem eine wichtige Studie über die Slaven in Niederösterreich (Šembera $1844 \mathrm{f}$.), eine fast 400 Seiten starke Monographie zu den Westslaven in der Urzeit (Šembera 1868) sowie eine aufwendig recherchierte, mit statistischem Zahlenmaterial gespickte Studie über die Tschechen, Mährer und Slovaken samt ihren Siedlungsgebieten (Šembera 1877). Ebensowenig wurden spätere Untersuchungen über die Tschechen in Niederösterreich wie jene von A. Hubka $(1899,1901)$ eingearbeitet.

Auch aus germanistischer Sicht könnte das Quellenmaterial noch ergänzt werden; wir denken z. B. gerade für das Waldviertel an die Liste von $307(+14)$ abgekommenen Siedlungen von Heinrich Weigl (1937), die mehrere eingedeutschte Namen slavischer Herkunft enthält, die sich nicht im Glossar befinden. Die Ortsnamenkarte des niederösterreichischen Waldviertels nach Karl Lechner im Anhang zu Stepan (1937), die u. a. slavische Ortsnamen, genetivische Ortsnamen nach slavischen Personennamen und zweifelhaft slavische Ortsnamen ausweist, sowie Lechners Beitrag zur Besiedlungs- und Herrschaftsgeschichte des Waldviertels (Lechner 1937) könnten ebenfalls gute Dienste für ein weiteres Quellenstudium leisten, denn selbst

${ }^{3}$ Der ehemals im Tschechischen benutzte Name Manhartská čtvrt bezog sich auf ein Viertel am Manhart, also geographisch nicht genau unterscheidend auf die nördlichen Teile des Wald- und Weinviertels (Hausner - Šrámek 1996: 400). 
deutschtümelnde Historiker konnten aufgrund der Faktenlage die ehemals auch slavische Besiedlung des Waldviertels nie leugnen. ${ }^{4}$

Schon die bloße Durchsicht einer Landkarte des Grenzgebiets hätte das Quellenmaterial vermehren können, vgl. z. B. die im Glossar fehlenden Ortsnamen von Dörfern wie Klein-Litschau, Radschin, Türnau u. a. im nördlichen Waldviertel. So bekannte Namen wie Dürnkrut $=$ č. slk. Suché Kruty $<*$ Chrъwati oder Weitra $=$ č. Vitoraz als möglicherweise possessivische Ableitung von *Vitoradb sucht man im Namenbuch überhaupt vergeblich. Bis heute in Gebrauch steht etwa č. Vitorazsko für das Weitraer Gebiet; gebräuchlich war auch č. Vitorazská cesta von Weitra bis Stift Zwettl in Verlängerung des seit dem Mittelalter für den Handel bedeutsamen Beheim- bzw. Windisch-Steigs (česká stezka) von Weitra nach Böhmen. Die Etymologie des Namens Weitra, für den in historischen tschechischen Quellen auch Vitrov zu finden ist, ist zwar nicht restlos geklärt, doch erscheint in diesem Fall - zumindest für die tschechische Bezeichnung - slavische Herkunft (vgl. auch unter *Witrb in Bergermayer 2005: 285-286) wahrscheinlicher als alle, bislang unbefriedigenden Deutungsversuche aus dem Mittelhochdeutschen (etwa als ursprünglicher Flussname < mhd. wìt und mhd. ahe; so schon bei Lechner 1937: 20). Dafür sprechen nicht nur die Erstbelege Witrah, Wìtrah, Widra, Witra um die Wende vom 12. zum 13. Jahrhundert oder die Nennung des Orts durch Josef Valentin Zlobický in Jungmann (1839/V: 113) als Weitrach, sondern die ebenfalls relativ früh eingedeutschten, ursprünglich slavischen Ortsnamen der näheren Umgebung wie Schagges < *Čakanъ (Bergermayer 2005: 51-52) oder Wultschau $<*$ W(ъ)lčı (Bergermayer 2005: 290).

Die Onomastik darf in ihrem Verständnis als eigene Wissenschaft nicht der Verlockung erliegen, sich vornehmlich auf sich selbst zu beziehen. Bei Bergermayers Buch gewinnt man ein wenig den Eindruck, dass für die Auswahl der in ihrem Glossar erklärten Etyma eher die bereits vorliegende und zweifellos wertvolle onomastische Fachliteratur von Bedeutung war als die kritische Auseinandersetzung mit verschiedensten Quellen unter Berücksichtigung der Erkenntnisse der modernen Kontaktlinguistik. So verwundert etwa bei der Auswahl der slavischen Vergleichsnamen, dass zwar mitunter selbst ausgefallene Namen vom Elb- und Ostseeslavischen bis ins Bulgarische angeführt, nahe liegende tschechische und - häufiger noch - slovakische Entsprechungen eingedeutschter Namen slavischer Herkunft in Niederösterreich jedoch übergangen werden, falls sie nicht in der verwendeten onomastischen Literatur bei Profous, Svoboda u. a. angeführt sind. In diesem Fall hätte z. B. für die Ortsnamen ein kurzer Blick in die aktuellen Ortsnamenregister tschechischer oder

4 „Die Tatsache der ohnehin nicht so dicht gewesenen Slawenbesiedlung unserer Heimat wird einen wirklich intelligenten, objektiv denkenden Menschen kaum beunruhigen; für die Vergangenheit kann niemand und für die Zukunft wird jeder deutsche Mann sorgen, daß unsere Heimat, die schon über ein halbes Jahrtausend vor den Slawen Germanengut war, auch fernerhin deutsch bleibt.“ (Hrodegh 1925: 104). 
slovakischer Autoatlanten rasch ein weitaus umfangreicheres Vergleichsergebnis gezeitigt.

Was die von Bergermayer (2005) genannte onomastische Fachliteratur selbst angeht, so ist diese aus slavistischer Sicht zu vervollständigen. In Bezug auf den beschriebenen Raum und den zweifellos wichtigen Vergleich mit den angrenzenden Regionen und Siedlungsräumen überrascht das Fehlen manch wichtiger Fachpublikation, insbesondere tschechischer und slovakischer Provenienz. Abgesehen von den schon erwähnten Arbeiten von Šembera 1844 f., 1868, 1877 und Hubka 1899, 1901 ergänzen wir an dieser Stelle nur Beneš 1998; Berger 1991/1992; Bergmann 2003; Bohdanecký 1910; Černý - Váša 1907; Cuřín 1967; Eichler 2000; Eichler - Šrámek 1988; Giesler 1997; Hladký 2004; Hosák - Krandžalov 1962; HSSJ 1991 ff. (das Wörterbuch des historischen Slovakischen bringt gerade für die älteste Zeit immer wieder Belege auch in Form von Toponyma und Anthroponyma, so etwa gleich für das erste bei Bergermayer angeführte Lemma *aworb als „,mons Iawor“ mit einem Beleg von 1244 aus der Zips; HSSJ I: 520); Kavuljak 1955; Kiss 1978; Kočářek Kočárek 1992; Kotyška 1895; Krško 2003; Lutterer 1969; Lutterer - Kropáček - Huňáček 1976; Lutterer - Majtán - Šrámek 1982; Macháček 1906; Majtán 1972; Malenínská 1994; Matúšová 1996; Moldanová 2004; Nekuda 1961; Olivová-Nezbedová - Malenínská 2000; Palacký 1848; Popowska-Taborska 1968; Pleskalová 1992; Roubík 1959; Rudolf 1981/82; Šafař́k 1837, 1843; Schwarz 1931, ${ }^{2} 1961$; Sedláček 1877, 1908, 1920; Šmilauer 1932, 1960, 1969; Šrámek 1999; Stanislav 1948; Steinhauser 1941; Varsik 1964 ff.; Vašek 1872; VSOS und Žigo 1999 u. a. m. ${ }^{5}$

Wenn auch nicht im unmittelbaren Zusammenhang mit dem eigentlichen Thema stehend, wäre es im Allgemeinen nicht nur aus slavistischer Sicht für alle Benützer eines solchen Wörterbuchs ein zusätzlicher Informationsgewinn gewesen, wenn in einem kurzen, ergänzenden Exkurs auch die früher gebrauchten, heute aber weithin unbekannten slavischen Exonyma für Orts-, Flur-, Berg- und Gewässernamen in Niederösterreich zumindest auszugsweise angeführt worden wären, selbst wenn die entsprechenden Etyma der heute gebräuchlichen Namen bloß vielleicht oder nur vermeintlich slavischer Herkunft sind bzw. für sie überhaupt keine slavische Herkunft postuliert werden kann. Eine solche ergänzende Übersicht wäre auch schon deshalb zu begrüßen, weil wir erst so einen Gesamteindruck über den in der Siedlungsgeschichte dokumentierten, tatsächlichen Sprach- und Kulturkontakt zwischen bairisch- und slavischsprachiger Bevölkerung erhalten. Außerdem kann im Falle von tatsächlich eingedeutschten Namen slavischer Herkunft die tschechische Entsprechung noch heute für die Etymologisierung der Etyma entscheidende Hinweise liefern.

Bergermayer (2005: 81-82) erklärt etwa den Namen der Siedlung Göllitzhof nahe Heinrichs bei Weitra (Gde. Unserfrau-Altweitra) trotz der Belege Gelitz in Un-

\footnotetext{
${ }^{5}$ Vgl. noch weitere Literatur in Newerkla 2004: 631-710.
} 
ser Frauen pharr (1496) und in der Gellitz (1499) sowie der Namensvariante Jölitzhof im Einklang mit HONB (III/1970: 191) und Schuster (II/1990: 338 J 34) aus slav. *Golica zu *gols 'kahl, bloß, nackt, unbehaart' (bzw. mit geringerer Wahrscheinlichkeit von slav. *Jalica zu *jals 'unfruchtbar, öde'). Für erstere Etymologie müsste sie also mit der Verkürzung einer Verbindung des Adjektivs * gola mit einem femininen Substantiv (z. B. *dolina 'Tal'), einer nachfolgenden Ableitung auf *-ica von *gola, einer Umlautung des -o- im Deutschen zu -ö- und nachfolgender Entrundung im 13. Jahrhundert zu $-e-$ rechnen. Die Wahrheit ist hingegen nahe liegender, und das im wahrsten Sinne des Wortes, nämlich 500 Meter jenseits der Grenze auf böhmischen Gebiet, wo der eigentliche, heute verödete Ort mit dem deutschen Namen Göllitz lag, nämlich das tschechische Jedlice ${ }^{6}<$ slav. *Edlica zu *edla 'Tanne' (Profous II/1949: 113), - ein durchaus treffender Name für ein Dorf im an Tannen reichen Gebiet am Fuße des Mandelsteinmassivs, auf den Bergermayer (2005: 7576) im Zusammenhang mit *edla sogar selbst verweist.

Aus oben genannten Gründen führen wir nun in einer knappen und unvollständigen Aufzählung die lediglich in einigen Fällen noch bis heute verwendeten, wichtigsten tschechischen (bzw. tschechisierten) Bezeichnungen für Toponyme, Hydronyme $(\approx \approx \approx)$, Oro- und Anoikonyme $(\Delta)$ auf dem historischen Gebiet von Niederösterreich an, und zwar ohne Rücksicht auf ihre tatsächliche Entstehungszeit oder Etymologie. ${ }^{7}$ Die mit Asteriskus (*) markierten Namen werden bei Bergermayer (2005) näher erklärt, fast immer jedoch ohne Anführung der tschechischen exonymischen Entsprechungen. In Kursivdruck stehen in den Namenkonkordanzen ${ }^{8}$ alle Exonyme aus heutiger Sicht, also auch die deutschen Bezeichnungen für historisch niederösterreichische Orte, die 1919 an die Tschechoslowakei abzutreten waren.

${ }^{6}$ Vgl. im Übrigen auch Gödelitz in Deutschland < altsorb. *Jedlica (Eichler I/1985: 146). Die lautliche Vereinfachung von $-d l->-l l-$ bzw. $-l-$ (z. B. tschech. sedlo 'Sattel' $>$ sello, selo) ist den tschechischen Dialekten in den böhmischen Randgebieten nicht unbekannt.

7 Es finden sich in den Aufstellungen also zum Teil auch spätere, nachträgliche oder falsch motivierte Neubildungen und Calquierungen. Nach der Angabe der Ortsnamen erfolgt in runder Klammer die Anführung der zugehörigen Gemeinde (sollte der Ort zugleich Hauptort einer Gemeinde sein, entfällt diese). Bei Gewässer-, Berg- und Flurnamen wird die Gemeinde nur dann genannt, wenn erstere einer einzigen Gemeinde eindeutig zugeordnet werden können.

${ }^{8}$ Unsere Sammlung der tschechischen Entsprechungen beruht auf Exzerpten u. a. aus Bohdanecký 1910; Garscha 1995; Hausner - Šámek 1996; Hubka 1899, 1901; Kočářek Kočárek 1992; Macháček 1906; Šafař́k 1837, 1842; Sedláček 1877; Šembera 1844 f., 1868, 1877 und Landkarten sowie Atlanten aus der Zeit vom 16. Jahrhundert (z. B. MM 2003, FKMM 2004) bis in die Anfangsjahre des 20. Jahrhunderts (z. B. Padalík 1880/83; Plesinger 1899; Plodek 1894; Šembera 1863, 1881; Wagner 1869, 1884, 1899, 1900). Vgl. auch die digitale Landkartenbibliothek der Védecká knihovna in Olomouc (Olmütz) unter http://mapy.vkol.cz/. Die Publikation von Beránek et al. 2006 erschien so knapp vor der Drucklegung dieses Beitrags, dass sie nicht mehr berücksichtigt werden konnte. 
Waldviertel - Viertel ober dem Manhartsberg (Nadmanhartsko $)^{9}$

Abbrand (vormals Gde. Rottenschachen): Spáleniště. Albrechtsberg an der Großen Krems: Albrechtsberk. Allentsgschwendt (Gde. Lichtenau im Waldviertel): Aloltskšvend. Allentsteig*: Alendšteig [-štajk]. Almosen (Gde. Göpfritz an der Wild): Almužna. Altenburg: Staré Hrady. Altpölla* (Gde. Pölla): Staré Polany. Altwaidhofen (Gde. Waidhofen an der Thaya): Starý Bejdov. Altweitra (Gde. Unserfrau-Altweitra): Starý Vitoraz. Äpfelgschwendt (Gde. Göpfritz an der Wild): Hepfenkšvend. Artolz (Gde. Pfaffenschlag bei Waidhofen an der Thaya): Artolec. Bad Großpertholz: Velký Pertolec (auch Velký Bertolec). Beinhöfen (č.): Německé ${ }^{10}$. Bernschlag (Gde. Allentsteig): Pernšlák. Blößberg ( $\Delta$, Gde. Irnfritz-Messern): Ples. Blumau an der Wild (Gde. Ludweis-Aigen): Blumava. Böhmhof (Gde. Zwettl / NÖ): České Dvory. Böhmsdorf (Gde. Groß Gerungs): Česká Ves. Böhmzeil (č.): Česká Cejle ${ }^{11}$. Bosnien (vormals Gde. Rottenschachen): Bosna. Brand (Gde. Brand-Nagelberg) (č.): Lomy. Braunaubach ( $\approx \approx)$ : Skřemelice (auch Branná, Brunava). Breiteneich (Gde. Horn): Široký $D u b$. Breitensee (Gde. Gmünd, vormals Gde. Zuggers): Bratisej. Breitl ( $\Delta$, Gde. RosenburgMold): Prádlo. Brunn (Gde. Waidhofen an der Thaya-Land): Brun. Brunn am Wald (Gde. Lichtenau im Waldviertel): Brun. Buchberg ( $\Delta$, Gde. Dietmanns): Buková hora. Buchberg am Kamp (Gde. Gars am Kamp): Buchberk. Burg Ranna (Gde. Mühldorf): Hrad Raná. Burgschleinitz* (Gde. Burgschleinitz-Kühnring): Hrad Slivnice. Dallein (Gde. Geras): Dolany. Deutsche Thaya* ( $\approx \approx)$ : Rakouská Dyje. Dietmanns: Ditmanec. Dimling* (Gde. Waidhofen an der Thaya): Dubnice. Doberndorf (Gde. Horn): Dobranov. Dobersberg*: Dobersberk. Dobra* (Gde. Pölla): Dobrá (auch Doubrava). Dobrabach* ( $\approx \approx)$ : Doubrava. Dobrawald* $(\Delta \text {, Gde. Pölla): les Doubrava. Dobratendorf (= Oberndorf, Gde. Pölla })^{12}$ : Dobrotín. Döbring ( $\Delta$, Gde. Ludweis-Aigen): Dobrnice. Dorf Rosenau (Gde. Zwettl / NÖ): Rožnov. Dreiländerstein ( $\Delta$, Gde. Dobersberg): Trojmezí. Drösiedl (Gde. Ludweis-Aigen): Drosidl. Drosendorf* (Gde. Drosendorf-Zissersdorf): Drozdovice. Droß: Drozd. Dürnstein: Suchý Kámen (auch Dürnštein [-štajn]). Edelbach (Gde. Allentsteig): Erlbach. Eggenburg: Imburk. Eichberg (Gde. Großdietmanns): Dub. Eichhorns (Gde. Pölla): Veveři. Eisenberg (Gde. Krumau am Kamp): Eisenberk [Ajzen-]. Eisgarn*: Našky. Els (Gde. Albrechtsberg an der Großen Krems): Olešná. Elsarn im Strassertal (Gde. Strass im Strassertale): Olšany. Elsern (Gde. Drosendorf-Zissersdorf): Olšany (auch Oslany). Engelbrechts (Gde. Kautzen): Engelbrechtice. Erdweis: Nová Ves nad Lužnicí (vormals Hlinná). Erdweiser Mooshäuser (vormals Gde. Erdweis): Záblatí. Erdwieser Waldhäuser (vormals Gde. Erdweis): Lesní Chalupy. Etzmannsdorf am Kamp (Gde. Gars am Kamp): Ecmansdorf. Etzmannsdorf bei Straning (Gde. Straning-Grafenberg): Ecmansdorf. Feistritz* (Gde. Raxendorf): Bystřice. Felling (Gde. Hardegg): Vrbno. Felsenberg (Gde. Pölla): Felsenberk. Fernitz* (Gde. Schönberg am Kamp): Borovnice. Finsternau (Gde. Brand-Nagelberg): Finsternava (auch Finsterov). Fischbachhäuser (vormals Gde. Zuggers): Rybné. Fischerhäuseln (vormals Gde. Weißenbach): Fischerovy Chalupy. Fistritz* (Gde. Groß-Siegharts): Bystřice. Flachau (Gde. Zwettl / NÖ): Vlachov. Fohra (Gde. Kottes-Purk): Borová. Franzen* (Gde. Pölla): Vrančí. Fratres* (Gde. Waldkirchen an der Thaya): Fratres (auch Rablin). Frattingbach $(\approx \approx \approx)$ : Lubnice. Frauenhofen (Gde. Sankt Bernhard-Frauenhofen): Vranov (auch Frauenhof). Freischling (Gde. Schönberg am Kamp): Frišlink. Friedersbach (Gde. Zwettl / NÖ): Fridršbach. Fronsburg (Gde. Weitersfeld): Fronsburk. Frühwärts (Gde. Gastern): Sekvaň. Fuglau* (Gde. Al-

${ }^{9}$ Bei jenen Waldviertler Orten, hinter denen in runder Klammer č. steht, sind tschechischsprachige Einwohner im 19. Jh. in entsprechender Anzahl belegt.

${ }^{10}$ Heute Ortsteil von Dvory nad Lužnicí.

11 Heute Straßenzug zum Zollhaus von Č̉ eské Velenice - Gmünd.

12 Bereits im 19. Jahrhundert abgekommene Siedlung. 
tenburg): Fukla. Fugnitz* (Gde. Geras): Fugnice. Fugnitz* ( $\approx \approx)$ : Fugnice. Gaberbach* $(\approx \approx \approx):$ Javorka. Gaberkirche* (Gde. Raabs an der Thaya): Javoři. Ganz (Gde. Schwarzenau): Jasany. Ganzberg ( $\Delta$, Gde. Schwarzenau): Mirky. Garmanns (Gde. Gföhl): Jermaneč. Gars*: Gorš (auch Garšs, Gars, Gors). Gastern*: Chrastná. Gehringsmühle (Gde. Waldkirchen an der Thaya): Jarovický mlýn. Georgenberg (Gde. Göpfritz an der Wild): Svatý Jiř́. Geras*: Jerouš (auch Jeruš). Gernitzbach ( $\approx \approx \approx)$ : Gegnice. Gföhl: Kvíl (auch Kvíle). Gilgenberg (Gde. Waldkirchen an der Thaya): Jilkov. Gloden (Gde. Lichtenau im Waldviertel): Kladno. Gmünd (č.): Cmunt. Goggitsch* (Gde. Geras): Kokač. Gollersbach ( $\approx \approx$, Gde. Pölla): Jeřice. Göllitzhof* (Gde. Unserfrau-Altweitra): Jedlice. Göpfritz an der Wild: Kypřice. Göpfritzschlag (Gde. Karlstein an der Thaya): Gotfrídec. Goritz ( $\Delta$, Gde. Geras): Buková hora. Goslarn* (Gde. Japons): Kozlany. Gradnitz* (Gde. Zwettl / NÖ): Hradnice. Grad-

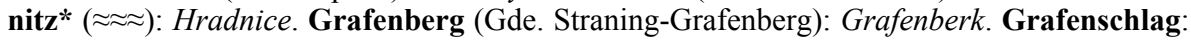
Grafenšlák. Gratzenbach $(\approx \approx \approx$, Gde. Unserfrau-Altweitra): Novohradský potok. Groß Burgstall (Gde. Sankt Bernhard-Frauenhofen): Purkštal. Groß Gerungs: Velké Kerušice. Großau* (Gde. Raabs an der Thaya): Hrazová (auch Grosava, Kraznová). Groß-Eberharts (Gde. Pfaffenschlag bei Waidhofen an der Thaya): Velký Eberharec. Große Krems $(\approx \approx \approx)$ : Křemže. Großer Radlbach $(\approx \approx \approx$, Gde. Waidhofen an der Thaya): Jesenice. Großgerharts (Gde. Thaya): Velký Kerhárec (auch Velké Kerhárce). Großglobnitz* (Gde. Zwettl / NÖ): Velká Hlohnice (auch Velká Glognice). Großgöttfritz: Velký Gotfrídec. Großhaselbach (Gde. Schwarzenau): Velký Haselbach. Großhaslau (Gde. Zwettl / NÖ): Velký Hazlov. Großmotten (Gde. Gföhl): Velký Otín. Großpoppen (Gde. Allentsteig): Velké Popovany. Groß-Radischen* (Gde. Eisgarn): Radešin (auch Velký Radešov). Großreinprechts (Gde. Lichtenau im Waldviertel): Reinprechtec [Rajn-]. Großrupprechts (Gde. Vitis): Ruprechtec. Großschönau: Velký Šenov (auch Velký Šonov). Groß-Siegharts: Velké Sieghartice (auch Sihorec, Zihorec, Velký Zigharec). Groß-Taxen (Gde. Kautzen): Velký Daksín. Großweißenbach (Gde. Großgöttfritz): Velké Višbachy (auch Velké Visbachy). Grünberg (Gde. Sankt Bernhard-Frauenhofen): Zelená Hora. Gundschachen (č.): Kunšach. Hardegg: Hardek (auch Hrádek). Harth (Gde. Geras): Harta (auch Varta). Haslau (Gde. Heidenreichstein): Hazlov. Heidenreichstein: Kamýk. Heinreichs (Gde. Pölla): Hendřiš. Hirschbühel ( $\Delta$, Gde. Raabs an der Thaya): Kozelec. Hirschenschlag (Gde. Reingers): Hirššlák. Hocheck ( $\Delta$, Gde. Waldhausen): les Javornice. Hohenau (Gde. Dobersberg): Vysoký Dub. Hoheneich: Vysoký Dub. Hollenbach (Gde. Waidhofen an der Thaya): Helbach. Horach $(\Delta$, Gde. Schönberg am Kamp): Hora. Horasberg ( $\Delta$ ): Hora. Hörmanns (Gde. Zwettl / NÖ): Herrmaneč. Hörmanns bei Litschau (Gde. Litschau): Heřmanec. Horn: Rohy (auch Roh). Hostiz ( $\Delta$, Gde. Raabs an der Thaya): Hoštice. Idolsberg (Gde. Krumau am Kamp): Idoltsberk. Illmau (Gde. Kautzen): Jilmová (auch Jilmo). Irnfritz (Gde. Irnfritz-Messern): Irnfriz. Jaidhof: Jadov (auch Jaidhof [Jajd-]). Japons*: Kostelni Japanice. Jarolden* (Gde. Thaya): Jarotín. Jasenegg* (Gde. Weiten): Jesenik. Jasnitz* (Gde. Waidhofen an der Thaya): Jesenice. Jaudling* (Gde. Vitis): Javornice. Jaudlingbach $(\approx \approx \approx)$ : Javornice. Jauerling* $(\Delta)$ : Javornice (auch Javorník). Jessen ( $\Delta$, Gde. Irnfritz-Messern): Jeseny. Jetzles (Gde. Vitis): Jecles. Joachimstal (Gde. Sankt Martin): Jáchymov. Josefschlag (vormals Gde. Böhmzeil): Žižkovo předměstí ${ }^{13}$ (vormals auch Josefovsko). Kainraths (Gde. Litschau): Kunratec. Kainraths (Gde. Waidhofen an der Thaya-Land): Kunratec. Kamegg: Kampek. Kamp ( $\approx \approx)$ : Kouba (auch Chuba, Kamp). Kattau* (Gde. Meiseldorf): Kadov. Kautzen: Choucno (auch Chocenice, Kaucno). Kaja* (Gde. Hardegg): Kyjov (auch Chýje). Kibitzhöfe* (Gde. Thaya): Divišùv dvưr. Kirchberg an der Wild (Gde. Göpfritz an der Wild): Kirchberk (auch Kiriberk). Kirchenberg ( $\Delta$, Gde. Straning-Grafenberg): Hưry. Kleineberharts (Gde. Waidhofen an der Thaya): Malý Eberharec.

${ }^{13}$ Heute Ortsteil von České Velenice. 
Kleiner Radlbach ( $\approx \approx \approx$, Gde. Waidhofen an der Thaya): Jesenice. Kleingerharts (Gde. Kautzen): Malý Kerhárec (auch Malé Kerhárce). Kleingloms (Gde. Vitis): Malá Hlohnice. Klein-Göpfritz (Gde. Pfaffenschlag bei Waidhofen an der Thaya): Malé Kyprice. Kleingöttfritz (Gde. Grafenschlag): Malý Gotfrídec. Kleinhaselbach (Gde. Allentsteig): Malý Haselbach. Kleinkainraths (Gde. Allentsteig): Kunratec. Klein-Litschau (Gde. Eggern): Malý Ličov. Klein-Meiseldorf* (Gde. Meiseldorf): Malý Mysliboř (auch Malé Myslibořice). Kleinotten (Gde. Zwettl / NÖ): Malý Otín. Kleinpertholz (Gde. Heidenreichstein): Malý Pertolec (auch Malý Bertolec). Kleinpoppen (Gde. Echsenbach): Malé Popovany. Kleinraabs (Gde. Pölla): Rakousky (auch Rakousy v Polanech). Klein-Radischen* (Gde. Eisgarn): Radešinek. Kleinschönau (Gde. Zwettl / NÖ): Malý Šenov (auch Malý Šonov). Klein-Taxen (Gde. Kautzen): Malý Daksín. Kleinweißenbach (Gde. Großgöttfritz): Malé Višbachy (auch Malé Visbachy). Kleinzwettl* (Gde. Gastern): Malá Světlá. Kloster Pernegg (Gde. Pernegg): Pernek (auch Horni Pernek). Klumetzberg ( $\Delta$, Gde. Raabs an der Thaya): Chlum (auch Chlumec). Koblhof (Gde. Zwettl / NÖ): Koblov. Kogelberg ( $\Delta$, Gde. Straning-Grafenberg): Jesinky. Koggendorf (Gde. Raabs an der Thaya): Kobeřice. Kollmitz ( $\Delta$, Gde. Weißenkirchen in der Wachau): Chlumec. Kollmitzberg ( $\Delta$, Gde. Raabs an der Thaya): Chlumec. Kollmitzdörfl (Gde. Raabs an der Thaya): Chlumec. Kollmitzgraben (Gde. Raabs an der Thaya): Chlumecké Zákopí. Kolm* (Gde. Melk): Chlum. Kolmberg (Gde. Senftenberg): Chlum. Kottaun* (Gde. Geras): Chodouny. Kottschallings (Gde. Windigsteig): Koštálov (auch Košt'álkov). Kotzendorf (Gde. Gars am Kamp): Kocendorf. Krems an der Donau (č.): Křemže (auch Kremže). Krems ( $\approx \approx \approx)$ : Křemže. Kronsegg (Gde. Langenlois): Kronsek. Krumau am Kamp: Krumlov. Lainsitz* ( $\approx \approx \approx)$ : Lužnice. Langau: Lanava (auch Lanova). Langenlois*: Dlouhá Louže. Laufenegg* (Gde. Raxendorf): Lubník. Lauterbach (Gde. Moorbad Harbach): Lautrpoch. Lehndorf (Gde. Pernegg): Levín. Leiben: Lipina. Leisberg (Gde. Gföhl): Lysá Hora. Leisberg ( $\Delta$, Gde. Gföhl): Lysá hora. Lengenfeld: Dlouhé Pole. Leopoldsdorf (Gde. Reingers): Lipoltice (auch Lipoltec). Lexnitz* (Gde. Dobersberg): Leštnička (auch Lešnice). Lexnitzbach* ( $\approx \approx)$ : Leštnice. Lichtenau im Waldviertel: Lichtnov. Liebnitz* (Gde. Raabs an der Thaya): Libenice. Lindau (Gde. Raabs an der Thaya): Lindava. Litschau*: Ličov. Litschgraben* (Gde. Gföhl): Lidče. Loibes* (Gde. Groß-Siegharts): Libuš. Loiwein* (Gde. Lichtenau im Waldviertel): Lubna (auch Lubno). Loisbach* ( $\approx \approx)$ : Louže. London (vormals Gde. Rottenschachen): Londýn (Velký L., Malý L.). Loschberg (Gde. Waldhausen): Lošberk. Luden* (Gde. Raabs an der Thaya): Ludeň (auch Lubnice). Ludweis (Gde. Ludweis-Aigen): Ludkovice (auch Ludwejš [-vajš]). Mährische Thaya* ( $\approx \approx)$ : Moravská Dyje. Maiersch* (Gde. Gars am Kamp): Mojžír (auch Marýž). Maißwald ( $\Delta$, Gde. Dobersberg + Gde. Karlstein an der Thaya): Kunice. Manhartsberg ( $\Delta)$ : Manhartský vrch (auch Lůna). Maria Dreieichen (Gde. Rosenburg-Mold): Tři Duby. Maria Laach am Jauerling: Marie na Luhu. Matzelsdorf (Gde. Burgschleinitz-Kühnring): Macelsdorf. Meires (Gde. Windigsteig): Mojžǐr (auch Marýž). Meiseldorf*: Mysliboř. Merkengersch (Gde. Dobersberg): Merkengerst. Messern (Gde. Irnfritz-Messern): Mesary. Modlisch* (Gde. Schwarzenau): Modlič. Modsiedl* (Gde. Raabs an der Thaya): Močidla. Mödring* (Gde. Horn): Modřice. Mödringbach $(\approx \approx \approx)$ : Modřice. Moidrams* (Gde. Zwettl / NÖ): Modřany. Mold (Gde. Rosenburg-Mold): Molt. Moorbad Harbach: Chorpach. Mörtersdorf (Gde. Rosenburg-Mold): Modrá. Morwitz ( $\Delta$, Gde. Horn): les Moravice. Nagelberg (č.) (Gde. Brand-Nagelberg): Nagelberk. Naglitz: Nakolice (vormals auch Nekalice). Nebelstein ( $\Delta$, Gde. Moorbad Harbach): Nebeštán. Neukirchen an der Wild (Gde. Brunn an der Wild): Nový Kostelec. Neupölla (Gde. Pölla): Nové Polany. Neuriegers (Gde. Raabs an der Thaya): Nová Ligeř. New York (vormals Gde. Schwarzbach): Nový York. Niederedlitz* (Gde. Thaya): Dolní Jedlice. Niederfladnitz* (Gde. Hardegg): Dolni Blatnice. Niederglobnitz* (Gde. Zwettl / NÖ): Dolni Hlohnice (auch Dolni Glognice). Niedergrünbach (Gde. Rastenfeld): Dolní Grünbach. Niedernondorf (Gde. Waldhausen): Dolní Nová Ves. Niederplöttbach (Gde. Pölla): Dolní Pletbachy. Niederranna* (Gde. Mühldorf): Dolní Raná. Niederschrems* (Gde. Schrems): Dolní 
Skřemelice. Niklasberg (Gde. Raabs an der Thaya): Svatý Mikuláš. Nondorf an der Wild (Gde. Irnfritz-Messern): Nová Ves. Nonndorf (Gde. Raabs an der Thaya): Nová Ves. Nonndorf bei Gars (Gde. Gars an der Thaya): Nová Ves. Oberedlitz* (Gde. Thaya): Horní Jedlice. Oberer Molder Berg ( $\Delta$, Gde. Rosenburg-Mold): Horni Modrá. Oberfladnitz* (Gde. Weitersfeld): Horní Blatnice. Obergrünbach (Gde. Karlstein an der Thaya): Horní Grünbach. Obergrünbach (Gde. Lichtenau im Waldviertel): Horni Grünbach. Oberhöflein (Gde. Weitersfeld): Hevlany (auch Horni Hevlín). Oberlainsitz* (Gde. Sankt Martin): Lužnice. Obermixnitz* (Gde. Weitersfeld): Horni Mišnice (auch Horni Miksnice). Oberndorf (Gde. Zwettl / NÖ): Horní Ves. Oberndorf bei Raabs (Gde. Raabs an der Thaya): Horní Ves Rakouská. Oberndorf bei Weikertschlag (Gde. Raabs an der Thaya): Horni Ves. Obernondorf (Gde. Waldhausen): Horní Nová Ves. Oberpfaffendorf (Gde. Raabs an der Thaya): Horní Pfaffendorf [Fafen-]. Oberplank (Gde. Schönberg am Kamp): Ranice. Oberplöttbach (Gde. Zwettl / NÖ): Horní Pletbachy. Oberranna* (Gde. Mühldorf): Horní Raná. Oberthumeritz* (Gde. Japons): Černé Tubeřice (auch Horni Tubeřice). Oberthürnau* (Gde. Drosendorf-Zissersdorf): Horní Trnávka. Ottenstein (Gde. Rastenfeld): Otenštein [-štajn]. Ostra (Gde. Weinzierl am Walde): Ostrá. Paris (vormals Gde. Rottenschachen): Paříž. Pengerswald ( $\Delta$, Gde. Dobersberg): les Sosny. Pernegg: Pernek. Piegerbach ( $\approx \approx \approx$, Gde. Geras): Pijavice. Plank am Kamp* (Gde. Schönberg am Kamp): Plaveník. Pleßberg* (Gde. Kautzen): Ples. Pölla*: Polany. Pölla* ( $\Delta$, Gde. Meiseldorf): Polany. Pöllahof (Gde. Raxendorf): Polanský dvuir. Pötzbach $(\approx \approx \approx$, Gde. Pölla): Police. Primmersdorf* (Gde. Raabs an der Thaya): Přdměřce (auch Př́ldmérice, Primeršdorf). Prutzendorf (Gde. Weitersfeld): Prucendorf: Prutzendorfer Bach $(\approx \approx \approx)$ : Olešná. Puch (Gde. Waidhofen an der Thaya): Buk. Purgstall (Gde. Geras): Purkštal na Lehnici. Pyhra (Gde. Waidhofen an der Thaya): Pirha (auch Laz). Raabs an der Thaya: Rakousy nad Dyjí. Raan (Gde. Schönberg am Kamp): Rovná (auch Rajná). Radessen* (Gde. Ludweis-Aigen): Radešín (auch Radouš). Radl* (Gde. Ludweis-Aigen): Rádlo. Radschin (Gde. Kautzen): Račín (auch Hradčany). Rappolz (Gde. Waldkirchen an der Thaya): Rapotín. Rappottenstein: Rapotenštein [-štajn]. Rastenberg (Gde. Rastenfeld): Rastberk (auch Rastenberk). Reibers (Gde. Dobersberg): Rejvír (auch Rejbiřr). Reichau* (Gde. Weinzierl am Walde): Rýha (auch Rejcha). Reichenau am Freiwald (Gde. Bad Großpertholz): Rychnov. Reichers (Gde. Großgöttfritz): Rejchýř. Reichharts (Gde. Irnfritz-Messern): Rejchárec. Reinberg-Dobersberg (Gde. Kautzen): Rimberk. Reinberg-Heidenreichstein (Gde. Eggern): Rimberk-Kamýk. Reinberg-Litschau (Gde. Eggern): Rimberk-Ličov. Reingers: Rankerr. Reinolz (Gde. Dobersberg): Rejnolce (auch Rejnolec). Reinprechtspölla (Gde. Burgschleinitz-Kühnring): Polany Reinprechtovy [Rajn-]. Reißbach ( $\approx \approx \approx)$ : Dračice (auch Kastanice, Kostenice). Reith (Gde. Raabs an der Thaya): Jesenice. Riegers (Gde. Dobersberg): Ligeř. Riegersburg (Gde. Hardegg): Rigrsburk. Riemerhof (Gde. Zwettl / NÖ): dvi̊r Ranice. Rodingersdorf* (Gde. Sigmundsherberg): Radikov. Röschitz*: Řešice. Rosenauer Wald ( $\Delta$, Gde. Zwettl / NÖ + Gde. Groß Gerungs): Rožnovský les. Rosenburg (Gde. Rosenburg-Mold): Rosenburk. Rossa* (Gde. Raabs an der Thaya): Rosochy. Rothweinsdorf* (Gde. Irnfritz-Messern): Radvanice. Rottenschachen (č.): Rapšach. Rudmanns (Gde. Zwettl / NÖ): Rudmanč. Rudolz (Gde. Waldkirchen an der Thaya): Rudolec. Sallapulka (Gde. Weitersfeld): Šalapulka (auch Salapulka). Sallingberg: Salingberk. Sankt Bernhard (Gde. Sankt Bernhard-Frauenhofen): Svatý Bernart. Sankt Leonhard am Hornerwald: Svatý Linhart. Sankt Marein (Gde. Brunn an der Wild): Panna Marie (auch Panna Maria). Sankt Martin: Svatý Martin. Sarmingbach $(\approx \approx \approx)$ : Žabnice. Saß (Obere S., Mittlere S., Untere S.) $(\Delta$, Gde. Geras): les Sosny. Saßwald ( $\Delta$, Gde. Thaya): les Zdislav. Sauggern* (Gde. Ludweis-Aigen): Suchá. Schacherdorf (Gde. Vitis): Šachov. Schaditz (Gde. Raabs an der Thaya): Šatice. Schandachen (Gde. Litschau): Šandachy. Schauenstein (Gde. Pölla): Šauenštein [-štajn]. Schellingshof (Gde. Dobersberg): dvưr Želenice. Schirmannsreith* (Gde. Geras): Širmansreut [-rajt]. Schirnes* (Gde. Thaya): Černice. Schirnfeld ( $\Delta$, Gde. Japons): Černé. Schiltern (Gde. Langenlois): Śtitáry. Schladeinbach $(\approx \approx \approx$, Gde. Karlstein an der Thaya): 
Slatinka. Schlader* (Gde. Karlstein an der Thaya): Slatinka (auch Slatina). Schlag (Gde. Schwarzenau): Šláky. Schloss Rosenau (Gde. Zwettl / NÖ): zámek Rožnov. Schloteinmühle $(\Delta$, Gde. Pölla): Slatinský mlýn. Schloteinbach ( $\approx \approx \approx$, Gde. Pölla): Slatinka. Schönau bei Litschau (Gde. Litschau): Šenava. Schönberg am Kamp: Šumberk. Schönfeld (Gde. Göpfritz an der Wild): Šumfeld. Schrems* (̌̌.): Skřemelice (auch Šremelice, Křemelice). Schwarzbach (č.): Tušt' (vormals Švarcbach). Schwarzenau: Švarcenava. Schwarzau (Gde. Moorbad Harbach): Švarcava. Schwarzaubach $(\approx \approx \approx)$ : Černá. Schlagles (Gde. Allentsteig): Šláčky. Seebs* (Gde. Ludweis-Aigen): Šébs (auch Seč). Selbitz (Gde. Rappottenstein): Selvice. Seyfrieds (Gde. Heidenreichstein): Žibridec. Sieghartsles (Gde. Groß-Siegharts): Malý Zigharec. Sigmundsherberg: Zikmundova. Sirnitzbach $(\approx \approx \approx)$ : Sirnice. Sofienwald = Sophienwald (vormals Gde. Erdweis): Žofina Hut'. Söllitz* (Gde. Pölla): Želice (auch Solice). Sommerleiten (Gde. Ludweis-Aigen): Dobrnice. Sprögnitz* (Gde. Großgöttfritz): Spretnice (auch Spretinec). Staningersdorf* (Gde. Pernegg): Staňkov (auch Stanikov). Starrein* (Gde. Weitersfeld): Stařin. Steinbach (Gde. Allentsteig): Štumbach. Steinbach (Gde. Brand-Nagelberg): Štumbach. Steinberg ( $\Delta$, Gde. Pfaffenschlag bei Waidhofen an der Thaya): les Trnava. Stiefern* (Gde. Schönberg am Kamp): Štivina (auch Štivnice). Stift Zwettl* (Gde. Zwettl / NÖ): Klášter Světlá (auch Klášter Světelský). Stockern (Gde. Meiseldorf): Stakory. Stoies* (Gde. Vitis): Stojanov. Stoitzenberg ( $\Delta$, Gde. Eggenburg): Stoječín. Stoitzendorf* (Gde. Eggenburg): Stoječín. Stranberg ( $\Delta$, Gde. Karlstein an der Thaya): Stráň. Straning* (Gde. Straning-Grafenberg): Stránky. Stranitzberg ( $\Delta$, Gde. Gars am Kamp): Stránce. Strass im Strassertale: Stráž (auch Stras). Strobnitzbach ( $\approx \approx \approx$, Gde. Moorbad Harbach): Stropnice. Strögen* (Gde. Sankt Bernhard-Frauenhofen): Střehom. Syrnau* (heute Teil von Zwettl / NÖ): Syrná (auch Sirná). Tabor ( $\Delta$, Gde. Gars am Kamp): Tábor. Tannenbruck (č.): Trpnouze ${ }^{14}$. Taubitz (Gde. Lichtenau im Waldviertel): Doubice (auch Dubnice). Teichwiesenbach $(\approx \approx \approx)$ : Bobova. Thaua* (Gde. Allentsteig): Tuchom. Thaures* (Gde. Heidenreichstein): Tur̆any. Thaures* (Gde. Pölla): Tur̆any. Thaya*: Dyje. Theras* (Gde. Sigmundsherberg): Teras. Thiergarten (vormals Gde. Tannenbruck): Obora. Thuma* (Gde. Karlstein an der Thaya): Tumava. Thumeritzbach ( $\approx \approx \approx):$ Tubeřička. Thunau am Kamp* (Gde. Gars am Kamp): Tunava. Thures* (Gde. Karlstein an der Thaya): Turovec. Thürneustift (Gde. Schönberg am Kamp): Neuštift [Naj-]. Tiefenbach (Gde. Kautzen): Hluboká. Tiefenbach (Gde. Krumau am Kamp): Hluboká. Töbernitzbach ( $\approx \approx \approx$, Gde. Pölla): Debrnice (auch Teprnice). Trandorf (Gde. Mühldorf): Dráchov. Triftriegel ( $\Delta$, Gde. Lichtenau im Waldviertel): les Šachov. Triglas (Gde. Kautzen): Triglaz. Tröbings* (Gde. Ludweis-Aigen): Třebenice. Türnau (Gde. Haugschlag): Trnávka. Ulrichschlag (Gde. Waidhofen an der Thaya): Matějovice (auch Matějovec). Unserfrau am Sand (Gde. Unserfrau-Altweitra): Matka Boži na Písku. Unterer Molder Berg ( $\Delta$, Gde. Rosenburg-Mold): Dolní Modrá. Untermixnitz* (Gde. Weitersfeld): Dolni Mišnice (auch Dolni Miksnice). Unterpertholz (Gde. Raabs an der Thaya): Dolni Pertolec (auch Pertolec, Dolni Bertolec). Unterpfaffendorf (Gde. Raabs an der Thaya): Dolni Pfaffendorf [Fafen-]. Unterranna* (Gde. Mühldorf): Raná. Unterthumeritz* (Gde. Japons): Tubeřice (auch Dolni Tubeřice, Tumrice). Unterthürnau* (Gde. Drosendorf-Zissersdorf): Dolni Trnávka. Vitis*: Biteš. Waidhofen an der Thaya: Český Bejdov (auch Bejdov nad Dyji). Waldhers (Gde. Waldkirchen an der Thaya): Valtír. Wartberg (Gde. Straning-Grafenberg): Vartberk. Weikertschlag an der Thaya (Gde. Raabs an der Thaya): Vejkartice (auch Vajkršlok). Weinern (Gde. Groß-Siegharts): Vinary. Weinpolz (Gde. Göpfritz an der Wild): Humpolec. Weinpolzer Berg = Ebinger $\operatorname{Holz}(\Delta$, Gde. Göpfritz an der Wild): Humpolecký

${ }^{14}$ Heute Ortsteil von Hranice. 
vrch. Weißenbach: Vyšné. Weitra: Vitoraz. Wielands (Gde. Großdietmanns) (č.): Velenice ${ }^{15}$ (auch Bělenec). Wienings (Gde. Groß-Siegharts): Minice. Wiesmaden (Gde. Gastern): Mláčov. Wildberg (Gde. Irnfritz-Messern): Vildberk. Wilhelmshof (Gde. Raabs an der Thaya): dvůr Vilimov. Windigsteig: Vindiššteig [-štajk]. Witschkoberg (č.): Halámky. Wohoskahäu$\boldsymbol{s e r}=$ Wochuska (̌̌.) (vormals Gde. Witschkoberg): Ochůzky. Wolfshof (Gde. Gars am Kamp): Vlči Dvory. Wurschenaigen* (Gde. Lichtenau im Waldviertel): Vršany. Zemmendorfer Berg ( $\Delta$, Gde. Raabs an der Thaya): Kozelec. Zettlitz* (Gde. Drosendorf-Zissersdorf): Sedlice (auch Sedlec). Zissersdorf* (Gde. Drosendorf-Zissersdorf): Čistějov (auch Cišlsdorf). Zöbing (Gde. Langenlois): Zebín. Zogelsdorf (Gde. Burgschleinitz-Kühnring): Coglsdorf. Zogelsdorfer Bach $(\approx \approx \approx)$ : Slatina. Zötting ( $\Delta$, Gde. Raabs an der Thaya): Jesenice. Zuggers:

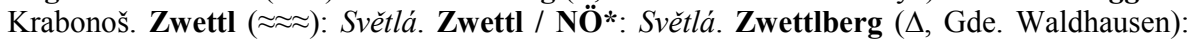
Světlá. Zwinzen* (Gde. Allentsteig): Svince (auch Svinec)

Weinviertel (Vinná čtvrt) - Viertel unter dem Manhartsberg (Podmanhartsko) ${ }^{16}$

Absdorf: Opatov. Altenbergen ( $\Delta$, Gde. Schrattenberg): Staré Hory. Altenmarkt (Gde. Gaubitsch): Starý Trh. Altenmarkt im Thale (Gde. Hollabrunn): Starý Trh v Dole. Althöflein (Gde. Großkrut): Starý Hevlín. Altlichtenwarth: Lichnibort (auch Lichtenvart). Alt-Prerau (Gde. Wildendürnbach): Starý Přerov. Altruppersdorf (Gde. Poysdorf): Starý Rupersdorf. Ameistal = Poybachtal ( $\Delta):$ Mravenči údolí. Angern an der March (kroat.): Congr. Andlersdorf (kroat.): Andlersdorf (auch Rozvrtňák). Asparn an der Zaya: Ošpra (auch Ošpry, Osikov, Osykov). Auggenthal (Gde. Haugsdorf): Augentál. Baumgarten am Wagram (Gde. Großweikersdorf): Pangort. Bergau (Gde. Göllersdorf): Pergov. Bernhardsthal (slk.): Pernitál. Bischofswarth (vormals Gde. Feldsberg) (kroat., slk.): Hlohovec. Blumenthal (Gde. Zistersdorf): Blumentál. Bockfließ: Puklis. Breitensee (Gde. Marchegg) (kroat.): Bratisej. Breitstetten (Gde. Leopoldsdorf im Marchfelde) (kroat.): Bratštatyn. Buchberg ( $\Delta$, Gde. Mailberg): Buková hora. Bullendorf (Gde. Wilfersdorf): Škarohlid (auch Škarohled). Deutsch-Wagram: Německý Ogrún (auch Hrubý Ogrún). Diepolz (Gde. Großharras): Děpolec. Dietersdorf (Gde. Hollabrunn): Ditersdorf. Dobermannsdorf* (Gde. Palterndorf-Dobermannsdorf): Dobrnašdorf (auch Obrnašdorf, Dobmanšdorf). Drasenhofen*: Drazovy Dvory. Drösing* (slk.): Střezenice (auch Střezemice). Dürnkrut (slk.): Suché Kruty (auch Suché Krupty). Ebenthal: Hrádek. Ebersbrunn (Gde. Hohenwarth-Mühlbach am Manhartsberg): Ebersbrun. Eggendorf am Walde (Gde. Maissau): Egendorf. Eggendorf im Thale (Gde. Hollabrunn): Egendorf. Eibesthal (Gde. Mistelbach): Eibestál [Ajbes-]. Eichenbrunn (Gde. Gnadendorf): Eichenbrun [Ajchen-]. Eichhorn (Gde. Zistersdorf): Ochorno. Engelhartstetten (kroat.): Poturno. Enzersdorf bei Staatz (Gde. Staatz): Enčesdorf. Enzersdorf im Thale (Gde. Hollabrunn): Enčisdorf v Dole. Erdberg (Gde. Poysdorf): Hrádek (auch Erdperk). Erdpress (Gde. Sulz im Weinviertel): Vemprisko. Ernsdorf bei Staatz (Gde. Staatz): Erensdorf (auch Erendorf). Ernstbrunn: Ernstbrun. Ernstbrunner Wald $(\Delta)$ : Ernstbrunský les. Fahndorf* (Gde. Ziersdorf): Fandorf. Falkenstein: Falkenštein [-štajn]. Fallbach: Falbach. Feldsberg (slk.): Valtice (vormals auch Valčice). Florianiberg ( $\Delta$, Gde. Ringelsdorf-Niederabsdorf): Capi hora. Föhrenwald ( $\Delta$, vormals Gde. Feldsberg): Boř́i les. Föllim (Gde. Poysdorf): Vrbovec. Franzensdorf (Gde. Groß-Enzersdorf) (kroat.): Kimišdorf. Frättingsdorf (Gde. Mistelbach): Vratikov. Friebritz (Gde. Fallbach): Fribrice. Fuchsenbigl (Gde. Haring-

15 Ein Teil des ehemaligen Unter-Wielands (Dolní Velenice, vormals auch Bělenec oder Běleč) gehört heute zu Ceské Velenice und somit zur Tschechischen Republik.

16 Bei jenen Orten, hinter denen in runder Klammer slk. bzw. kroat. steht, sind slowakischbzw. kroatischsprachige Einwohner in entsprechender Anzahl noch bis ins 19. Jh. belegt, bei manchen Orten wie z. B. Hohenau zum Teil sogar bis heute. 
see) (kroat.): Fuchspichl. Füllersdorf (Gde. Großmugl): Filersdorf. Fünfkirchen (Gde. Drasenhofen): Pětikostelí. Furth (Gde. Göllersdorf): Furt. Gaiselberg (Gde. Zistersdorf): Geiselberk [Gajzel-]. Garschönthal* (vormals Gde. Feldsberg) (slk.): Úvaly (ehemals auch Garšentál). Gaubitsch*: Kováč. Gaweinstal (vormals Gaunersdorf)*: Gauneršdorf (auch Gaunesdorf). Gebmanns (Gde. Ernstbrunn): Gebmanč. Gettsdorf (Gde. Ziersdorf): Gecdorf. Ginzersdorf (Gde. Großkrut): Ginčesdorf. Glaubendorf (Gde. Heldenberg): Klaubendorf. Gnadendorf: Hnanice. Goggendorf (Gde. Sitzendorf an der Schmida): Kokendorf. Golitschberg ( $\Delta$, Gde. Retz): Holič. Göllersdorf: Gelesdorf. Gösing am Wagram* (Gde. Fels am Wagram): Jeseník. Gösting* (Gde. Zistersdorf): Kostniky (auch Kestink). Götzendorf (Gde. Velm-Götzendorf): Vrbovec. Grafensulz (Gde. Ladendorf): Grafensulce (auch Sulec). Grenzschlössl (vormals Gde. Feldsberg): Hraniční zámeček. Groißenbrunn (Gde. Engelhartstetten): Kisibrun. Groß-Enzersdorf: Hrubý Enčisdorf. Großharras: Velké Harasy. Groß-Inzersdorf (Gde. Zistersdorf): Velký Enčisdorf. Groß-Kadolz (Gde. SeefeldKadolz): Velký Kadolec. Großkrut*: Slovenské Kruty (auch České Kruty, České Krupty). Großmeiseldorf* (Gde. Ziersdorf): Mysliboř. Großmugl: Velké Mohelno. Großnondorf (Gde. Guntersdorf): Nová ves. Großriedenthal: Velký Riedentál. Großrußbach: Velký Rusbach. Großstelzendorf (Gde. Göllersdorf): Velký Štelcendorf. Großweikersdorf*: Velký Weikersdorf [Vajkrs-]. Großwetzdorf (Gde. Heldenberg): Velký Vecdorf. Grund (Gde. Wullersdorf): Grunt. Guttenbrunn (Gde. Ottenthal): Gutenbrun. Hadres: Hadrasy (auch Hedresy). Haidhof (vormals Gde. Feldsberg): dvůr Ovčárna. Hanfthal (Gde. Laa an der Thaya): Hanftál. Haringsee (kroat.): Horisej. Haugsdorf: Hauksdorf. Hausbrunn: Hasprun (auch Hazbrun, Hasbrun). Hauskirchen: Haukskirch. Herrnbaumgarten: Panský Pangort (auch Panské Dubenky, Panský Pangrot). Herrnleis (Ged. Ladendorf): Panská Lysá (auch Panská Lisa). Herzogbirbaum (Gde. Großmugl): Herzogbirnbaum. Höbersbrunn (Gde. Gaweinstal): Hebertsbrun. Hocheck ( $\Delta$, Gde. Niederleis): Hůry. Hochleithenwald $(\Delta)$ : $H o-$ lajka. Hohenau an der March (slk.): Cahnov. Hohenwarth (Gde. Hohenwarth-Mühlbach am Manhartsberg): Hohenvart. Hollabrunn (vor 1928 Oberhollabrunn): Horni Holabrun (auch Horni Holobrón). Hollenstein (Gde. Ziersdorf): Holštein [-štajn]. Hörersdorf (Gde. Mistelbach): Heroltsdorf. Hüttendorf (Gde. Mistelbach): Hitendorf. Im Greut ( $\Delta$, Gde. Heldenberg): Luky. Jedenspeigen: Nidršpelk. Jetzelsdorf (Gde. Haugsdorf): Ičlsdorf (auch Jeclsdorf). Kalladorf (Gde. Wullersdorf): Chvalov (auch Kaladorf). Karlhof (Gde. Großharras): Karlov. Katzelsdorf (Gde. Bernhardsthal): Kačisdorf. Kettlasbrunn (Gde. Mistelbach): Ketesbrun (auch Kelesbrun). Ketzelsdorf (Gde. Poysdorf): Keclsdorf. Kirchstetten (Gde. Neudorf bei Staatz): Kirchštetín. Kleedorf (Gde. Hollabrunn): Kledorf. Kleinhadersdorf (Gde. Poysdorf): Hedrsdorf. Kleinhaugsdorf (Gde. Haugsdorf): Malý Hauksdorf. Kleinhöflein (Gde. Retz): Hevlínek. Kleinkadolz (Gde. Hollabrunn): Malý Kadolec. Kleinriedenthal (Gde. Retz): Malý Riedentál. Kleinschweinbarth (Gde. Drasenhofen): Malý Svinvart. Kleinstelzendorf (Gde. Hollabrunn): Malý Štelcendorf. Kleinstetteldorf (Gde. Hollabrunn): Malý Štetldorf. Kleinweikersdorf (Gde. Nappersdorf-Kammersdorf): Malý Weikersdorf [Vajkrs-]. Kleinwetzdorf (Gde. Heldenberg): Malý Vecdorf. Kiblitz (Gde. Ziersdorf): Kublice. Kopfstetten (Gde. Eckartsau) (kroat.): Guštatyn. Korneuburg: Korneuburk. Kuchlwald ( $\Delta$, Gde. Staatz): Slanice. Kühberg (Gde. Maissau): Kraví hora. Laa an der Thaya: Lava. Ladendorf: Londorf (auch Ladendorf). Langenzersdorf: Dlouhý Enčisdorf. Lanzendorf (Gde. Mistelbach): Lancendorf. Lassee: Losej. Latschenberg $(\Delta)$ : Lačenberk. Leiser Berge $(\Delta)$ : Lysá hora. Limberg (Gde. Maissau): Lemberk (auch Limberk). Linienbachleiten ( $\Delta$, Gde. Neudorf bei Staatz): Kamenice. Loidesthal (Gde. Zistersdorf): Litultstál. Loimersdorf (Gde. Engelhartstetten) (kroat.): Limišdorf. Loosdorf* (Gde. Fallbach): Losdorf. Mailberg: Mailberk. Maisbirbaum (Gde. Ernstbrunn): Maisbirnbaum. Maissau*: Maisava. Mannersdorf an der March (Gde. Angern an der March) (kroat.): Magrsdorf. Mannsdorf an der Donau (kroat.): Selce. Marchegg (kroat.): Marchek. Maria Roggendorf* (Gde. Wullersdorf): Rukendorf. Matzen (Gde. Matzen-Raggendorf): Macno (auch Mačno). Michelstetten (Gde. As- 
parn an der Zaya): Michelštetín. Mistelbach: Myslibach (auch Mistelbach). Mittergrabern (Gde. Grabern): Prostředni Graben. Mitterretzbach (Gde. Retzbach): Prostřední Recbachy. Mottschüttel $(\Delta$, Gde. Großharras): Močidla. Mühlbach $(\approx \approx \approx$, Gde. Drasenhofen + Gde. Poysdorf): Mlýnský potok. Naglern (Gde. Ernstbrunn): Naglary. Neudorf bei Staatz: Nová Ves. Neuhof (vormals Gde. Feldsberg): Nový dvůr. Neuruppersdorf (Gde. Wildendürnbach): Nový Rupersdorf. Neusiedl an der Zaya: Najsigl (auch Neusidl). Niederabsdorf (Gde. Ringelsdorf-Niederabsdorf): Dolni Opatov (auch Abšdorf). Niederfellabrunn (Gde. Niederhollabrunn): Dolni Felabrun. Niederhollabrunn: Dolni Holabrun. Niederkreuzstetten (Gde. Kreuzstetten): Dolní Kreuzštetín [Krajc-]. Niederleis*: Dolní Lysá (auch Dolní Li$s a$ ). Niederrußbach (Gde. Rußbach): Dolni Rusbach. Niederschleinz* (Gde. Sitzendorf an der Schmida): Dolní Slivnice. Niedersulz (Gde. Sulz im Weinviertel): Dolní Sulce. Nursch* (Gde. Großmugl): Nuš. Oberdürnbach (Gde. Maissau): Horní Dürnbach. Oberfellabrunn (Gde. Hollabrunn): Horni Felabrun. Obergrabern (Gde. Grabern): Horní Graben. Oberhof (Gde. Seefeld-Kadolz): Horní dvi̊r. Oberkreuzstetten (Gde. Kreuzstetten): Horní Kreuzštetín [Krajc-]. Oberleis* (Gde. Ernstbrunn): Horni Lysá (auch Horní Lisa). Obermarkersdorf (Gde. Schrattenthal): Horni Markersdorf. Obernalb* (Gde. Retz): Horní Nalb. Oberparschenbrunn* (Gde. Göllersdorf): Horni Pašenbrun. Oberretzbach (Gde. Retzbach): Horní Recbachy. Oberrußbach (Gde. Rußbach): Horni Rusbach. Oberschoderlee (Gde. Stronsdorf): Šatrle. Obersulz (Gde. Sulz im Weinviertel): Hrubé Sulce (auch Horní Sulce, Horní Sulec). Oberstinkenbrunn (Gde. Wullersdorf): Horni Štinkenbrun. Oberthemenau (vormals Gde. Feldsberg) (kroat., slk.): Charvátská Nová Ves. Oberthern* (Gde. Heldenberg): Horní Trnava. Oberweiden (Gde. Weiden an der March): Horní Weiden [Vajd ${ }^{\circledR} \mathrm{n}$. Obritz (Gde. Hadres): Ubrice (auch Olbrechtec). Orth an der Donau (kroat.): Ort. Ottenthal: Otnitál (auch Odnitál). Paasdorf (Gde. Mistelbach): Pasdorf. Palterndorf (Gde. Palterndorf-Dobermannsdorf): Paltramdorf. Passauerhof (Gde. Poysdorf): Pasovský dvirr. Patzenthal (Gde. Strohnsdorf): Pacentál. Patzmannsdorf (Gde. Strohnsdorf): Pacmansdorf. Pellendorf (Gde. Gaweinstal): Pelendorf. Pernhofen-Gutshof (Gde. Laa an der Thaya): Pernov. Pfaffstetten (Gde. Ravelsbach): Pfafštetín [Faf-]. Pframa (Gde. Eckartsau) (kroat.): Fráma. Platt (Gde. Zellerndorf): Plat. Pollitzerberg ( $\Delta$, Gde. Hadres): Police. Porrau* (Gde. Göllersdorf): Borová. Porrauer Bach ( $\approx \approx$, Gde. Göllersdorf): Borová. Pottenhofen (Gde. Wildendürnbach): Potenhof. Poysbrunn* (Gde. Poysdorf): Pušbrun. Poysdorf*: Pušdorf. Prottes*: Protes. Puch (Gde. Hollabrunn): Buk (auch Bok). Pulkau*: Pulkava (auch Puklava). Pulkau* ( $\approx \approx)$ : Pulkava. Pürstendorf (Gde. Ladendorf): Pirstendorf. Pustaberg ( $\Delta$, Gde. Falkenstein): Pustá. Pyhra (Gde. Gnadendorf): Pirha. Rabensburg (slk.): Ranšpurk (auch Havranohrad). Radlbrunn* (Gde. Ziersdorf): Radenbrun. Rafing* (Gde. Pulkau): Revink. Raistenberg ( $\Delta$, Gde. Schrattenberg): Rajstna. Raschala* (Gde. Hollabrunn): Rašala. Reintal (Gde. Bernhardsthal): Rentál. Retz*: Rec (auch Rejc, Reteč, Řič). Retz-Altstadt: Staré město. Ringelsdorf (Gde. Ringelsdorf-Niederabsdorf) (slk.): Lingašdorf. Rohrbach (Gde. Ziersdorf): Rorbach. Russbach $(\approx \approx \approx)$ : Rusava. Sankt Ulrich (Gde. Neusiedl an der Zaya): Svatý Oldŕich. Schatzberg $(\Delta$, Gde. Seefeld-Kadolz): Šac. Schleinzbach $(\approx \approx \approx)$ : Slivnička. Schletz* (Gde. Asparn an der Zaya): Šlec. Schlosshof (Gde. Engelhartstetten): Zámeček. Schmida $(\approx \approx \approx)$ : Smidava. Schöngrabern (Gde. Grabern): Kravaře. Schönkirchen (Gde. SchönkirchenReyersdorf): Šenkarka (auch Šenkirch, Šenkirich). Schrattenberg: Šratenberk (auch Šatniberk). Schrattenthal: Šratentál. Schrick (Gde. Gaweinstal): Šrik. Seefeld (Gde. Seefeld-Kadolz): Séfeld. Sierndorf an der March* (Gde. Jedenspeigen) (slk.): Zindorf (auch Cíndorf). Sitzendorf an der Schmida: Sicendorf. Sonnberg (Gde. Hollabrunn): Žumberk. Spannberg: Šponberk. Spillern: Spilina. Staatz*: Stožec (auch Štác). Steinbergen ( $\Delta$, Gde. Schrattenberg): Skalky. Steinebrunn (Gde. Drasenhofen): Šteinabrun [Štajna-]. Stockerau: Štokrava. Straning Markt* (Gde. Straning-Grafenberg): Stránky. Straudorf (Gde. Haringsee) (kroat.): Štraudorf. Stronegg* (Gde. Stronsdorf): Stránek (auch Stranek). Stronsdorf*: Stransdorf. Stuhlberg (Gde. Gnadendorf): Chlumec. Sulz im Weinviertel: Sulce. Sulzbach ( $\approx \approx \approx):$ Sulce. 
Theimhof (vormals Gde. Feldsberg): Boří dvůr. Theimwald ( $\Delta$, vormals Gde. Feldsberg): Obora. Übermaiß ( $\Delta$, Gde. Falkenstein): les Roháč. Ungerndorf (Gde. Laa an der Thaya): Uherská Ves. Unterdürnbach (Gde. Maissau): Dolní Dürnbach. Untermarkersdorf (Gde. Hadres): Dolni Markersdorf. Unternalb* (Gde. Retz): Dolni Nalb. Unterparschenbrunn* (Gde. Sierndorf): Dolní Pašenbrun. Unterretzbach (Gde. Retzbach): Dolní Recbachy. Untersiebenbrunn (kroat.): Dolní Siebenbrun [Zíb ${ }^{2}$-]. Unterstinkenbrunn: Dolní Štinkenbrun. Unterthemenau (vormals Gde. Feldsberg) (kroat., slk.): Poštorná. Unterthern* (Gde. Heldenberg): Horni Trnava. Viendorf* (Gde. Göllersdorf): Vindorf. Wagram an der Donau (Gde. Eckartsau) (kroat.): Chorvatský Ogrún. Waidendorf (Gde. Dürnkrut): Vandorf. Waltersdorf an der March (Gde. Drösing) (slk.): Př́lepy. Walterskirchen (Gde. Poysdorf): Valkerskirch. Wartberg (Gde. Straning-Grafenberg): Vartberk. Watzelsdorf (Gde. Zellerndorf): Vecelsdorf. Wetzelsdorf (Gde. Poysdorf): Veclsdorf. Weidenbach ( $\approx \approx$ ): Vrbovka. Wetzleinsdorf (Gde. Großrußbach): Vecleinsdorf [Veclajns-]. Weyerburg (Gde. Hollabrunn): Wayerburk [Vajer-]. Wildendürnbach: Divoký Dürnbach. Wilfersdorf: Bilvašdorf (auch Bilvasdorf). Windisch Baumgarten (Gde. Zistersdorf) (kroat.): Pangort. Wischathal (Gde. Göllersdorf): Višetál. Wittau (Gde. Groß-Enzersdorf): Vitava. Wulzeshofen (Gde. Laa an der Thaya): Vlči Dvory (auch Vlči Hofy). Würnitz* (Gde. Harmannsdorf): Virnice. Zaya $(\approx \approx \approx)$ : Sajava. Zellerndorf: Celderndorf. Ziersdorf*: Čisdorf. Zistersdorf*: Čistějov. Zuckermandln ( $\Delta$, Gde. Schrattenberg): Homole. Zwerndorf* (Gde. Weiden an der March) (kroat.): Cvendorf. Zwingendorf (Gde. Großharras): Cvingendorf

Viertel ober dem Wienerwald (Čtvrt nad Videňským lesem)

Böheimkirchen: Český Kostelec. Edlitz* (Gde. Weinburg): Jedlice. Enns ( $\approx \approx \approx)$ : Enže. Erlauf $(\approx \approx \approx)$ : Erlafa (auch Erlava). Erlaufsee $(\approx \approx \approx)$ : Erlafské jezero. Fladnitz* $(\approx \approx \approx)$ : Blatnice. Gaming*: Kameník (auch Jamnice). Glocknitzhof* (Gde. Neulengbach): Glognice. Göblitz* (Gde. Haag): Kobylice. Golling an der Erlauf: Golnik. Großsierning* (Gde. Haunoldstein): Sirnice. Jeßnitz* ( $\approx \approx)$ : Jesenice. Jeßnitzhof* (Gde. Sankt Anton an der Jeßnitz): Jesenice. Kiritzerbach ( $\approx \approx \approx$, Gde. Furth bei Göttweig): Kyrice. Kleine Erlauf $(\approx \approx \approx)$ : Erlafka Kleinwien (Gde. Furth bei Göttweig): Videň. Kollmitz (Gde. Ardagger): Chlumec. Kollmitzberg (Gde. Ardagger): Chlumec. Kolm (Gde. Bergland): Chlum. Kremnitzbach* $(\approx \approx \approx)$ : Křemnice. Lonitzberg* (Gde. Steinakirchen am Forst): Lonice. Luegbach (vormals Feistra*) $(\approx \approx \approx)$ : Bystrá. Mautern an der Donau: Mutárny. Melk: Medlik. Oberwölbling (Gde. Wölbling): Horní Wölblink [Velb-]. Opponitz*: Opotnice. Perschling* (Gde. Weißenkirchen an der Perschling): Prstník (auch Prstnice). Perschling* ( $\approx \approx \approx)$ : Brznice (auch Prstnice). Pielach* (Gde. Melk): Bělá. Pielach* ( $\approx \approx)$ : Bèlá. Pöchlarn: Pechlary (auch Pechlarn). Preuwitz* (Gde. Zwentendorf an der Donau): Pribice. Ranzenbach* ( $\approx \approx \approx)$ : Radvanice. Raming* ( $\approx \approx \approx):$ Rudnice. Rems (Gde. Sankt Valentin): Remz. Rossatz (Gde. Rossatz-Arnsdorf): Rosatec. Salza $(\approx \approx \approx)$ : Salice. Sankt Andrä vor dem Hagenthale (Gde. Sankt Andrä-Wördern): Svatý Ondřej. Sankt Peter in der Au: Svatý Petr. Sankt Pölten: Svatý Hypolit. Sankt Valentin: Svatý Valentin. Sattel (Gde. Hollenstein an der Ybbs): Sedlo. Schachau (Gde. Oberndorf an der Melk): Šachov. Schloss Schallaburg (Gde. Schollach): Skála. Schlatten* (Gde. Ruprechtshofen): Slatinka. Sierning* (Gde. Bischofstetten): Sirnice. Sierning* ( $\approx \approx \approx):$ Sirnice. Stift Göttweig* (Gde. Furth bei Göttweig): Kotvik (auch Klášter Kotvík). Ströbitz* (Gde. Sankt Valentin): Stroblice. Ströblitz* (Gde. Wieselburg): Stroblice. Traisen ( $\approx \approx \approx)$ : Trejsma. Traismauer: Trýzma (auch Traismaur). Tulln: Dolany (auch Dolina). Türnitz: Trnovice. Waidhofen an der Ybbs: Bavorský Bejdov. Weistrach: Bystrá. Windischendorf (Gde. Euratsfeld): Slovanská Ves. Windischendorf (Gde. Ferschnitz): Slovanská Ves. Ybbs an der Donau: Ips (auch Jivice). Ybbs $(\approx \approx \approx)$ : Ybisa (auch Ipise). Ybbsitz*: Ipsice. Zaglau (Gde. Sankt Peter in der Au): Cáhlov. Zaucha* ( $\approx \approx):$ Suchá. Zauchbach* ( $\approx \approx \approx):$ Suchá 
Viertel unter dem Wienerwald (Čtvrt pod Videňským lesem)

Au am Leithagebirge (kroat.): Cindrov. Bad Deutsch-Altenburg: Staré Hrady. Baden: Rakouské Teplice. Bruck an der Leitha: Most nad Litavou. Edlitz*: Jedlice. Edlitzbach* $(\approx \approx \approx)$ : Jedlice. Feistritz am Wechsel*: Bystřice. Gablitz*: Kaplice (auch Jablice). Gloggnitz*: Glognice (auch Hlohnice). Göstritz* (Gde. Schottwien): Kostrice. Hainburg an der Donau: Hainburk. Haslau an der Donau (Gde. Haslau-Maria Ellend) (kroat.): Chorvatský Hazlov. Heiligenkreuz: Svatý Křžz. Hof am Leithagebirge (kroat.): Cimov. Höflein: Holavajna. Hradschin ( $\Delta$, Gde. Heiligenkreuz): Hradčany. Klosterneuburg: Klosterneuburk. Kulm* (Gde. Warth): Chlum. Kulmberg* ( $\Delta$, Gde. Warth): Chlum. Laab im Walde: Lúb. Landegg (Gde. Pottendorf) (kroat.): Landek. Mannersdorf am Leithagebirge (kroat.): $M a$ neršdorf. Markt Piesting*: Pístnice (auch Pístov, Pistnice). Mödling: Medlik. Moosbrunn: Malín. Neunkirchen: Nový Kostelec. Ödlitz* (Gde. Berndorf): Jedlice. Pernitz*: Pernice. Piesting* $(\approx \approx \approx)$ : Pistnice (auch Pistnice). Pischelsdorf (Gde. Götzendorf an der Leitha) (kroat.): Pišelsdorf. Pitten: Putiny. Pottenstein: Potštýn. Prigglitz: Priglice. Raglitz (Gde. Ternitz): Raglice. Reichenau an der Rax: Rychnov. Sankt Veit an der Triesting (Gde.

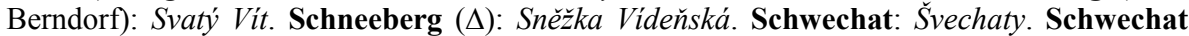
$(\approx \approx \approx)$ : Švechata. Sedlitzerhof (Gde. Klosterneuburg): Sedlice. Semmering*: Semerink. Semmering* $(\Delta)$ : Semernik (auch Semerink). Sommerein: Somorja. Steinfeld $(\Delta)$ : Kamenné pole. Steyersberg (Gde. Warth): Štejersberk. Tagnitz-Au ( $\Delta$, Gde. Ebenfurth): luh Tajnice. Ternitz*: Trnice (auch Ternice). Traiskirchen*: Traiskirch. Triesting* ( $\approx \approx \approx)$ : Trstnice. Trumau: Trumava. Weibnitz (Gde. Wartmannstetten): Vidnice. Wiener Neustadt: Videňské Nové Město. Wildungsmauer (Gde. Scharndorf) (kroat.): Vildungsmauer

\section{Wien (Videň)}

Am Spitz (21. Bez. Floridsdorf): Špice. Aspern (22. Bez. Donaustadt): Ošpra (auch Ošpry). Augarten ( $\Delta$, 2. Bez. Leopoldstadt): Lužánky. Breitenlee (22. Bez. Donaustadt): Prádlo. Döbling* (19. Bez.): Döblink [Deb-] (vormals Doblice). Favoriten (10. Bez.): Favority. Fünfhaus (15. Bez. Rudolfsheim-Fünfhaus): Pětidomy. Gaudenzdorf (12. Bez. Meidling): Gaudencdorf. Inzersdorf (23. Bez. Liesing): Inčisdorf (auch Inčersdorf). Jedlesee (21. Bez. Floridsdorf): Jedlisej. Kahlenberg ( $\Delta, 19$. Bez. Döbling): Lysá hora. Leopoldau (21. Bez. Floridsdorf): Břestová. Leopoldstadt (2. Bez.): Leopoldov. Liesing* (23. Bez.): Lesnice (auch Leštnice). Liesing* (auch Dürre Liesing) $(\approx \approx \approx)$ : Lesnice. Maria am Gestade $(\Delta, 1$. Bez. Innere Stadt): Panna Marie na Nábřeži. Meidling (12. Bez.): Meidlink [Majd-]. Oberlaa (10. Bez. Favoriten): Horní Lava. Ottakring (16. Bez.): Otakrink. Rodaun* (23. Bez. Liesing): Radouň (vormals Radúň). Sechshaus (15. Bez. Rudolfsheim-Fünfhaus): Šestidomy. Simmering (11. Bez.): Simerink. Stadlau (22. Bez. Donaustadt): Stadlava. Süßenbrunn (22. Bez. Donaustadt): Cisibrun. Unterlaa (10. Bez. Favoriten): Dolní Lava. Währing* (18. Bez.): Währink [Vérink]. Weidling (14. Bez. Penzing): Vídnice. Wieden (4. Bez.): Videňka

A bkürzungen

AK-NÖ 1864 ff.: Administrativ-Karte von Nieder-Oesterreich. Herausgegeben vom Verein für Landeskunde von Nieder-Oesterreich unter der Leitung des k. k. Rathes A. Steinhauser im Maße 1 Zoll $=400$ Wr. Klafter oder 1:28 800, 111 Sektionen, Wien 1864-1881 (Nachdruck 1961)

Beneš 1998: J. Beneš, Německá př́íjmení u Čechů (= Acta Universitatis Purkynianae 34, Slavogermanica IX.), 2 Bde., Ústí nad Labem [Rez. S. M. Newerkla, Wiener Slavistisches Jahrbuch 46 (2000) 339-340]

Beránek et al 2006:T. Beránek et al., Index českých exonym. Standardizované podoby, varianty. Index of Czech exonyms. Standardized forms, variants, Praha 
Berger 1991/1992: T. Berger, Tschechische Bezeichnungen für ausländische Ortsnamen mit besonderer Berücksichtigung von Bayern, Blätter für oberdeutsche Namensforschung 28/29, 77-123

Bergermayer 2005: A. Bergermayer, Glossar der Etyma der eingedeutschten Namen slavischer Herkunft in Niederösterreich (= Schriften der Balkan-Kommission 44), Wien

Bergmann 2003: H. Bergmann, Slawisches im Namengut der Osttiroler Gemeinden Ainet und Schlaiten: Anmerkungen zur Slavia submersa im vorderen Iseltal, Klagenfurt

Bína 2004: D. Bína, Idea regionu a pravděpodobnost vymezení Vitorazska, Výběr $41 / 3,183-190$

Bohdanecký 1910: E. Bohdanecký, Místní slovník slovanský v odcizovaných i cizích krajích, Praha

Brunner 1937: O. Brunner, Die geschichtliche Stellung des Waldviertels, in: Stepan 1937: 368-431

Černý - Váša 1907: F. Černý - P. Váša, Moravská jména místní. (Výklady filologické), Brno

Cuř́n 1967: $\quad$ F. Cuř́n, Studie z historické dialektologie a toponomastiky Čech, Praha

Czoernig 1857: K. J. Czoernig, Ethnographie der österreichischen Monarchie, 3 Bde., Wien

Eichler I/1985: $\quad$ E. Eichler, Slawische Ortsnamen zwischen Saale und Neiße. Ein Kompendium, I: A-J, Bautzen

Eichler 2000: $\quad$ E. Eichler, Zur Untersuchung der slavischen Toponyme in Nordbayern, in: K. Trost (ed.), Deutsch-tschechische Sprachbeziehungen. Germanismen, Personennamen, Ortsnamen (= SELP, Series II: Studia minora, Tom. VI), Regensburg, 85-95 [Rez. S. M. Newerkla, Wiener Slavistisches Jahrbuch 47 (2001) 266-269]

Eichler - Šrámek 1988: E. Eichler - R. Šrámek (eds.), Strukturtypen der slawischen Ortsnamen. Strukturní typy slovanské oikonymie, Leipzig

Ficker 1869: $\quad$ A. Ficker, Die Völkerstämme der Oesterreichisch-Ungarischen Monarchie, ihre Gebiete, Gränzen und Inseln. Historisch, geographisch und statistisch dargestellt, Wien

FKMM 2004: $\quad$ Fabriciovy a Komenského mapy Moravy. The Map of Moravia by Fabricius and Comenius. Die Landkarte Mährens von Fabricius und Komenský, CD-ROM, Praha - Přerov

Garscha 1995: $\quad$ W. R. Garscha, Hranice a jejich posuny. Rakousko-československá hranice 1918-1945, in: A. Komlosyová - V. Bůžek - F. Svátek (eds.), Kultury na hranici. Jižní Čechy - Jižní Morava - Waldviertel - Weinviertel, Wien, 69-74

Giesler 1997: J. Giesler, Der Ostalpenraum vom 8. bis zum 11. Jahrhundert. Studien zu archäologischen und schriftlichen Zeugnissen. Teil 2: Historische Interpretation (= Frühgeschichtliche und provinzialrömische Archäologie 1), Rahden

Gutkas 1990: K. Gutkas (Hg.), LandesChronik Niederösterreich. 3000 Jahre in Daten, Dokumenten und Bildern, Wien - München

Hausner - Šrámek 1996: I. Hausner - R. Šrámek, Glossar: Ortsnamen und ihre Herkunft, in: A. Bartoněk - B. Beneš - W. Müller-Funk - F. Polleross (eds.), Kulturführer Waldviertel - Weinviertel - Südmähren, Wien, 383-402

Hladký 2004:

J. Hladký, Hydronymia povodia Nitry, Trnava

Holzer 2001:

G. Holzer, Die Slaven im Erlaftal. Eine Namenlandschaft in Niederösterreich (= Studien und Forschungen aus dem Niederösterreichischen Institut für Landeskunde 29), Wien

HONB III/1970: H. Weigl, Historisches Ortsnamenbuch von Niederösterreich. III: H, I, Y, $\mathrm{J}, \mathrm{K}, \mathrm{Ch}$, Wien

Hosák - Krandžalov 1962: L. Hosák - D. Krandžalov, Souvislost slovanského místního názvosloví na Moravě a severovýchodních Bavořích, Časopis společnosti přátel starožitností 70, 92-101

Hrodegh 1925: $\quad$ A. Hrodegh, Das Waldviertel, 2. Band: Urgeschichte, Wien 
HSSJ:

Hubka 1899:

Historický slovník slovenského jazyka, Bratislava $1991 \mathrm{ff}$.

Hubka 1901:

A. Hubka, Naše menšiny a smíšené kraje na českém jihu, Praha

A. Hubka, Čechové v Dolních Rakousích. Studie z cest (= Knihovna Samostatnosti - Sbírka poučných spisů a pojednání o otázkách časových sv. 7), Praha

Jungmann: $\quad$ J. Jungmann, Slovník česko-německý, 5 Bde., Praha 1835-1839

Kavuljak 1955: A. Kavuljak, Historický miestopis Oravy, Bratislava

Kiss 1978:

L. Kiss, Földrajzi nevek etimológiai szótára [Etymologisches Wörterbuch der geographischen Namen], Budapest

Kočářek - Kočárek 1992: E. Kočárek sen. - E. Kočárek jun., Mühl- a Waldviertel, Novohradské hory, Český Krumlov, Třeboň a okolí. Průvodce, historické pohlednice (= Toulky př́hraničím, sv. 1), Plzeň

Kotyška 1895: $\quad$ V. Kotyška, Úplný místopisný slovník království Českého, Praha

Kronsteiner 1981: O. Kronsteiner, Die alpenslawischen Personennamen (= Österreichische Namenforschung, Sonderreihe 2), Wien

Krško 2003: $\quad$ J. Krško, Hydronymia povodia Turca, Banská Bystrica

Lechner 1937: K. Lechner, Besiedlungs- und Herrschaftsgeschichte, in: Stepan 1937: 3266

Lutterer 1969: I. Lutterer, Vývoj místních jmen a osídlení v povodí Orlic (= Knižnice Orlického muzea 6), Choceň

Lutterer - Kropáček - Huňáček 1976: I. Lutterer - L. Kropáček - V. Huňáček, Původ zeměpisných jmen, Praha

Lutterer - Majtán - Šrámek 1982: I. Lutterer - M. Majtán - R. Šrámek, Zeměpisná jména Československa. Slovník vybraných zeměpisných jmen s výkladem jejich původu a historického vývoje, Praha

Macháček 1906: J. Macháček, Territorialní vývoj a změny hranic Rakous, Korutan, Štyrska a Kraňska od r. 976-1250, in: Třicátá čtvrta výroční zpráva c. k. českého gymnasia v Českých Budějovicích za školní rok 1906, České Budějovice, 1-20 [+ 1 Karte im Anhang]

Majtán 1972: $\quad$ M. Majtán, Názvy obcí na Slovensku za ostatných dvesto rokov, Bratislava

Malenínská 1994: J. Malenínská, Zur Übernahme tschechischer Bergnamen ins Deutsche. (Am Material der Oronymie des Böhmischen Mittelgebirges), Onomastica Slavogermanica 21, 123-131

Matúšová 1996: J. Matúšová, Ortsnamen in mehrsprachigen Ländern und Regionen: deutsch/slavisch, in: E. Eichler et al. (eds.), Namenforschung. Ein internationales Handbuch zur Onomastik, Berlin, 1420-1426

MM 2003: $\quad$ Mapy Moravy ze 16.-18. století. The 16th to 18th century maps of Moravia. Die Landkarten Mährens aus der Zeit von 16. bis 18. Jahrhundert [P. Fabricius, J. A. Comenius, G. M. Vischer Tyrolensis, J. Ch. Müller], CDROM, Brno - Přerov

Moldanová 2004: D. Moldanová, Naše př́ijmení, Praha ${ }^{2} 2004$ [1983]

Nekuda 1961: V. Nekuda, Zaniklé osady na Moravě v období feudalismu, Brno

Newerkla 2004: S. M. Newerkla, Sprachkontakte Deutsch - Tschechisch - Slowakisch. Wörterbuch der deutschen Lehnwörter im Tschechischen und Slowakischen: historische Entwicklung, Beleglage, bisherige und neue Deutungen (= Schriften über Sprachen und Texte 7), Frankfurt am Main - Wien

Olivová-Nezbedová - Malenínská 2000: L. Olivová-Nezbedová - J. Malenínská (eds.), Slovník pomístních jmen v Čechách. Úvodní svazek, Praha

Padalík 1880/83: F. Padalík, Morava a Slezsko s částmi pohraničními Čech, Rakous, Uher a Pruska, 2 listy, Jihlava s. a. [zwischen 1880 und 1883]

Palacký 1848: $\quad$ F. Palacký, Popis království Českého čili podrobné poznamenání všech dosavadních krajův, panství, statkův, měst, městeček a vesnic, někdejších hradův a tvrzí, též samot a zpustlých osad mnohých v zemi české, s udáním jejich obyvatelstva dle popisu r. 1843, Praha

Plesinger 1899: V. Plesinger, Morava a Slezsko s pohraničními částmi Čech, Rakous, Uher a Pruska. Měřítko 1:200 000, 4 listy, Karlín 
Pleskalová 1992: J. Pleskalová, O tvoření českých pomístních jmen, Slovo a slovesnost 53, 193-200

Plodek 1894: $\quad$ K. Plodek, Církevní mapa biskupství brněnského dle nejspolehlivějších pramenů zeměpisných kreslil a vydává vlastním nákladem P. Kristin Plodek. Na vlastním skladě v Metličanech u Nového Bydžova (v Čechách). V komisi papežské tiskárny benediktínů Rajhradských v Brně (na Petrově), Brno

Popowska-Taborska 1968: H. Popowska-Taborska, Dawne podziały językowe zgermanizowanych terenów zachodniosłowiańskich, Onomastica 13, 142-163

Profous I/II/III: $\quad$ A. Profous, Místní jména v Čechách. Jejich vznik, původní význam a změny, I: A-H, Praha 1947; II: CH-L, Praha 1949; III: M- ̌́, Praha 1951

Profous - Svoboda 1957: A. Profous - J. Svoboda, Místní jména v Cechách. Jejich vznik, původní význam a změny, IV: S-Ž, Praha

Roubík 1959: $\quad$ F. Roubík, Soupis a mapa zaniklých osad v Čechách, Praha

Rudolf 1981/82: R. Rudolf, Zur deutschen Ortsnamenkunde in der Slowakei, Südostdeutsches Archiv 24/25, 9-18

Šafařík 1837: $\quad$ P. J. Šafařík, Slovanské starožitnosti, Praha

Šafařík 1843: $\quad$ P. J. Šafařík, Slavische Alterthümer, Leipzig

Schuster II/1990: E. Schuster, Die Etymologie der niederösterreichischen Ortsnamen. II: Ortsnamen F-M, Wien

Schwarz 1931, ${ }^{2}$ 1961: E. Schwarz, Die Ortsnamen der Sudetenländer als Geschichtsquelle, München - Breslau 1931, München - Berlin ${ }^{2} 1961$ [Rez. J. Janko, Časopis pro moderní filologii 21 (1935) 334-340]

Sedláček 1877: A. Sedláček, Jak se měnily a ustálily meze Čech a Rakous Dolních, in: Výroční zpráva cís. král. vyššího realného gymnasia $\mathrm{v}$ kr. městě Táboře za školní rok 1877, Tábor, 3-16

Sedláček 1908: A. Sedláček, Místopisný slovník historický království Českého, Praha

Sedláček 1920: $\quad$ A. Sedláček, Snůška starých jmen, jak se nazývaly v Čechách řeky, potoky, hory a lesy, Praha

Šembera 1844 f.: $\quad$ A. V. Šembera, O Slovanech v Dolních Rakousích, Časopis Českého museum 18 (1844), 536-549, 19 (1845), 163-189, 346-357 [inkl. Landkarte Osady slovenské a chorvatské v Dolnich Rakousích a u jezera Neziderského am Ende des zweiten Bandes des 19. Jahrgangs]

Šembera 1863: A. V. Šembera, Mapa země Moravské. S částmi pohraničnými Slezska, Čech, Rakous i Uher, 4 listy [ca. 1:288 000], Vídeň

Šembera 1868:

A. V. Šembera, Západní Slované v pravěku, Vídeň

Šembera 1877:

A. V. Šembera, Mnoho-li jest Čechů, Moravanů a Slováků v Rakousích a kde obývají. Příspěvek k ethnografii československé (Otištěno z Časopisu Musea král. Českého, 1876), Praha

Šembera 1881: A. V. Šembera, Mapa země Moravské. S částmi pohraničnými Slezska, Čech, Rakous i Uher, 4 listy [ca. 1:288 000], Vídeň

Šmilauer 1932: V. Šmilauer, Vodopis starého Slovenska, Praha - Bratislava

Šmilauer 1960: V. Šmilauer, Osídlení Čech ve světle místních jmen, Praha

Šmilauer 1969:

V. Šmilauer, Atlas místních jmen v Čechách, Praha

Soukup 1872:

Šrámek 1999:

J. N. Soukup, Výlet do Čech, Rakous a Solnohradu. Náčrty cestopisné. Druhé vydání (= Moravská bibliotéka XV), Velké Meziříčí

Stanislav 1948:

R. Srámek, Úvod do obecné onomastiky, Brno

Stepan 1937:

J. Stanislav, Slovenský juh v stredoveku, 2 Bde., Martin

E. Stepan (Hg.), Das Waldviertel, 7. Band: Geschichte, Wien

Steinhauser 1941: W. Steinhauser, Die Bedeutung der Ortsnamen in Niederdonau. II. Teil: Nordburgenland, St. Pölten - Wien

Svoboda 1964: J. Svoboda, Staročeská osobní jména a naše př́ijmení, Praha

Svoboda - Šmilauer 1960: J. Svoboda - V. Šmilauer, Místní jména v Čechách. Jejich vznik, původní význam a změny, V. Dodatky, Praha

Varsik 1964 ff.: $\quad$ B. Varsik, Osídlenie Košickej kotliny, 3 Bde., Bratislava 1964-1977

Vašek 1872:

A. Vašek, Výklad slovanských jmen na Opavsku, Programm des Gymnasiums zu Troppau 1872, 9-27 
VSOS: $\quad$ Vlastivedný slovník obcí na Slovensku, 3 Bde., Bratislava 1976-1978

Wagner 1869: J. E. Wagner, Cestovní mapa Čech, Moravy, Slezska, Rakous Horních i Dolních a zemí pohraničních. Měřítko 1:1 000 000, Praha

Wagner 1884: J. E. Wagner, Železniční a silniční mapa království Českého obsahující veškerá města, městyse a místa průmyslová. S naznačením veškerých cukrovarů a pivovarů. V měřítku 1:525 000. Se seznamem veškerých stanic poštovních, železničních, telegrafních a parolodí. S uvedením příslušných c. $\mathrm{k}$. okresních hejtmanství a c. k. okresních soudů u jednotlivých stanic $\mathrm{v}$ království Českém, Praha

Wagner 1899: $\quad$ J. E. Wagner, Cestovní mapa Čech, Moravy, Slezska, Rakous Horních i Dolních a zemí pohraničních. Měŕítko 1:1000 000. Do poslední doby doplněné nové vydání, Praha

Wagner 1900: J. E. Wagner, Jihovýchodní Čechy. Měřítko 1:220 000. Páté doplněné vydání, Praha

Weigl 1937: $\quad$ H. Weigl, Abgekommene Siedlungen, in: Stepan 1937: 277-279

Žigo 1999: $\quad$ P. Žigo, Hydronymia povodia Ipl'a, Bratislava

Stefan Michael Newerkla

Institut für Slawistik der Universität Wien

Universitätscampus AAKH, Hof 3

Spitalgasse 2, A-1090 Wien

stefan.newerkla@univie.ac.at 